\title{
The physiological basis for the computation of direction selectivity in the Drosophila OFF pathway
}

\author{
Giordano Ramos-Traslosheros ${ }^{1,2}$ and Marion Silies ${ }^{1^{*}}$ \\ ${ }^{1}$ Institute of Developmental Biology and Neurobiology, Johannes-Gutenberg University Mainz, 55128 Mainz, \\ Germany. \\ ${ }^{2}$ International Max Planck Research School Neuroscienes and Göttingen Graduate School for Neurosciences, \\ Biophysics, and Molecular Biosciences (GGNB) at the University of Göttingen \\ *e-mail: msilies@uni-mainz.de
}

\section{Abstract}

In Drosophila, direction-selective neurons implement a mechanism of motion computation similar to cortical neurons, using contrast-opponent receptive fields with $\mathrm{ON}$ and OFF subunits. It is not clear how the presynaptic circuitry of direction-selective neurons in the OFF pathway supports this computation, because all major inputs are OFF-rectified neurons. Here, we reveal the biological substrate for motion computation in the OFF pathway. Three interneurons, Tm2, Tm9 and CT1, also provide information about $\mathrm{ON}$ stimuli to the OFF direction-selective neuron $\mathrm{T}_{5}$ across its receptive field, supporting a contrast-opponent receptive field organization. Consistent with its prominent role in motion detection, variability in Tm9 receptive field properties is passed on to $\mathrm{T}_{5}$, and calcium decrements in $\mathrm{Tm} 9$ in response to $\mathrm{ON}$ stimuli are maintained across behavioral states, while spatial tuning is sharpened by active behavior. Together, our work shows how a key neuronal computation is implemented by its constituent neuronal circuit elements to ensure direction selectivity. 


\section{Introduction}

The algorithms by which neuronal circuits detect motion using the visual input from photoreceptors is widely considered a paradigmatic neural computation. Similar computations to achieve direction selectivity, a hallmark of motion detection, appear to be implemented in visual circuitry as diverse as the Drosophila visual system and the vertebrate retina. ${ }^{1-3}$ The neuronal circuits that extract direction-selective signals have been described in exquisite detail in Drosophila, perhaps the system in which we are closest to achieving a complete understanding of a neural computation from algorithm to circuitry and physiology..$^{4-6}$ However, despite recent work demonstrating that the Drosophila visual system implements a linear mechanism to compute motion, ${ }^{7}$ the implementation of this computation by the neuronal circuit elements has yet to be elucidated.

In Drosophila, direction selectivity emerges in the dendrites of $\mathrm{T}_{4}$ and $\mathrm{T}_{5}$ neurons, which respond to moving contrast increments $(\mathrm{ON})$ and decrements $(\mathrm{OFF})$, respectively. ${ }^{8,9}$ These neurons are at least three synapses away from photoreceptors and receive inputs from distinct lamina and medulla interneurons of the ON and OFF pathways. ${ }^{10-13}$ Two models, and their variants, have been used to describe direction-selective calcium signals in $\mathrm{T}_{4} / \mathrm{T}_{5} \cdot{ }^{10,14^{-17}}$ These rely on nonlinear operations that either amplify signals moving in the detector's preferred direction ${ }^{18}$ or suppress signals moving in the detector's null direction. ${ }^{19}$ However, recent evidence based on electrophysiology and voltage imaging argues that direction selectivity can also emerge from linear summing of $\mathrm{T}_{4} / \mathrm{T}_{5}$ inputs, ${ }^{7}$ or nonlinearly through sublinear integration in a portion of the $\mathrm{T}_{4} / \mathrm{T}_{5}$ receptive fields resulting in null-direction suppression. ${ }^{20,21}$ The resulting voltage from both models is then followed by a nonlinear voltage-to-calcium transformation. ${ }^{17}$ Specifically, sublinear voltage models are supported by experiments showing that direction-selective voltage signals in $\mathrm{T}_{4} / \mathrm{T}_{5}$ can be generated by summing synaptic input currents arising from individual bar responses of one contrast polarity. ${ }^{20,21}$ In turn, a linear model predicted $\mathrm{T}_{5}$ voltage responses to moving sine wave gratings. ${ }^{7}$ Furthermore, a dynamic nonlinearity in $\mathrm{T}_{4} / \mathrm{T}_{5}$ could adjust between these integration regimes. ${ }^{2}$

$\mathrm{ON}$ and OFF stimuli in the same location of the $\mathrm{T}_{5}$ receptive field produce voltage responses similar in amplitude, but opposite in sign. ${ }^{7}$ This highlights the requirement for integration of ON and OFF stimuli across the $\mathrm{T}_{5}$ receptive field. However, the cellular substrates that support this linear summation in $\mathrm{T}_{5}$ have not been described. Such a linear mechanism for direction selectivity parallels the one employed by simple cells in the vertebrate cortex of different species. ${ }^{23-26}$ The receptive fields of simple cells in primate cortex have two subunits with spatially opponent inhibition, i.e. for the same contrast polarity one subunit is excitatory and the other is inhibitory. ${ }^{27,28}$ Furthermore, cortical simple-cell receptive fields have a push-pull structure, meaning that at each receptive field location, inversion of the contrast polarity evokes a response of the opposite polarity, e.g. where ON stimuli provide excitation, OFF stimuli provide inhibition. ${ }^{27,29}$ Similarly, $\mathrm{T}_{4} / \mathrm{T}_{5}$ neurons have putative excitatory (depolarizing) and inhibitory (hyperpolarizing) receptive field subunits that each show contrast-opponent responses, i.e. opposite responses to ON and OFF contrasts. ${ }^{7,17,20,21,30}$ Thus direction-selective cells in the fly show contrast-opponent properties that resemble the push-pull structure of cortical simple-cell receptive fields. ${ }^{27}$ Simple cell receptive fields are a consequence of the synaptic inputs received from the lateral geniculate nucleus (LGN) relay neurons as well as from other cortical cells, and $\mathrm{T}_{4} / \mathrm{T}_{5}$ receptive fields are set up by their presynaptic medulla cell inputs. Ultimately, to determine the physiological basis of linear summation in motion computation, ${ }^{7}$ the $\mathrm{ON}$ and OFF inputs to the direction-selective neurons need to be characterized. 
Physiological studies of $\mathrm{T}_{4}$ and $\mathrm{T}_{5}$ response properties proposed that direction selectivity relies on spatiotemporal inputs from three points in space (Fig. $1 \mathbf{a}, \mathbf{b}) .{ }^{15,16,31}$ In both ON and OFF pathways, the medulla neurons that provide synaptic input to either the base or the tip of the dendrites have slower dynamics, whereas the central input is faster, consistent with the notion that direction selectivity requires temporal comparison of signals (Fig. $1 \mathbf{a}, \mathbf{b}) \cdot{ }^{10,32,33}$ Whereas $\mathrm{T}_{4}$ incorporates inputs from both, neurons that respond to $\mathrm{ON}$ and from neurons that respond to OFF signals, all major $\mathrm{T}_{5}$ inputs have been reported to be OFF rectified (Fig. ${ }_{1}$ b). ${ }^{10,31-36}$ Matching anatomy with the axis of motion, $\mathrm{T}_{5}$ receives its main visual inputs along the preferred motion direction axis from Tm9 on the trailing site, $\mathrm{Tm}_{1}, \mathrm{Tm}_{2}$ and $\mathrm{Tm}_{4}$ centrally, and $\mathrm{CT}_{1}$ on the leading site, with $\mathrm{CT}_{1}$ again mainly receiving Tm9 synaptic input (Fig. $\left.{ }_{1} \mathbf{b}\right) .{ }^{13}$ However, ON inputs are required to achieve the push-pull receptive field organization of $\mathrm{T}_{5}$, such that the excitatory subunit responds positively to OFF and negatively to ON, whereas the inhibitory subunit responds positively to ON and negatively to $\mathrm{OFF}^{7}$ (Fig. $1 \mathbf{c}$ ). Thus, it is not clear how $\mathrm{T}_{5}$ can receive the ON inputs required for its direction-selective receptive field.

Here, we investigate $\mathrm{ON}$ inputs, i.e. neurons that carry information about contrast increments, to $\mathrm{T}_{5}$ direction-selective cells in the OFF pathway to determine the cellular basis for the implementation of direction selectivity in $\mathrm{T}_{5}$ neurons. In vivo two-photon microscopy, dual-color calcium imaging, and characterization of all major OFF pathway interneurons reveal that Tm9, Tm2, and CT1 provide ON information to direction selectivity in the OFF pathway. Thus, Tm9, Tm2, and CT1 provide the basis for the $\mathrm{T}_{5}$ contrast-opponent receptive field subunits together with other OFF pathway neurons. Focusing on T5's major input, we show that receptive field properties of Tm9 are variable in size, and this variability correlates with $\mathrm{T}_{5}$ properties, thus demonstrating that $\mathrm{Tm} 9$ shapes downstream computations. Finally, we test whether these properties are stable across behavioral states, and whether motion computation relies on the same input properties. Mimicking an active behavioral state by activating octopamine receptors shows that both Tm9 variability and ON receptive field are maintained, whereas the Tm9 spatial receptive field is sharpened. Together, these findings reveal the cellular substrate of motion computation in the Drosophila OFF pathway and highlight the concrete neuronal properties that are responsible for direction selectivity. 
a

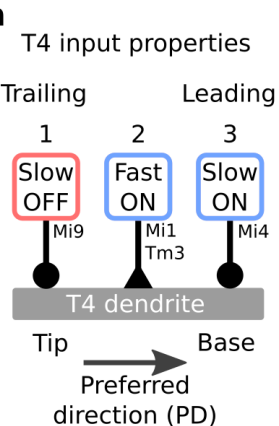

d

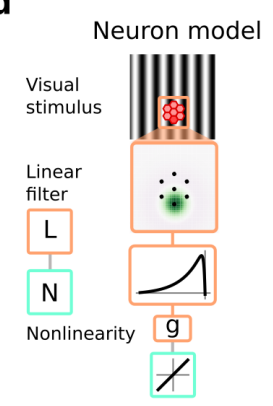

b

T5 input properties

Trailing Leading

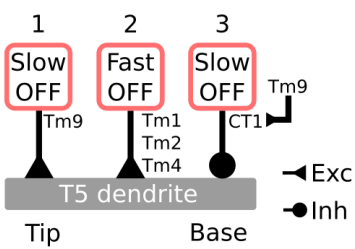

Base

$\rightarrow$ Inh

C

T5 voltage receptive field

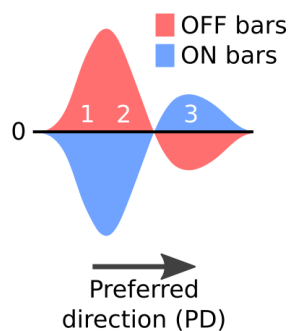

e Model of dendritic responses of T5 neurons

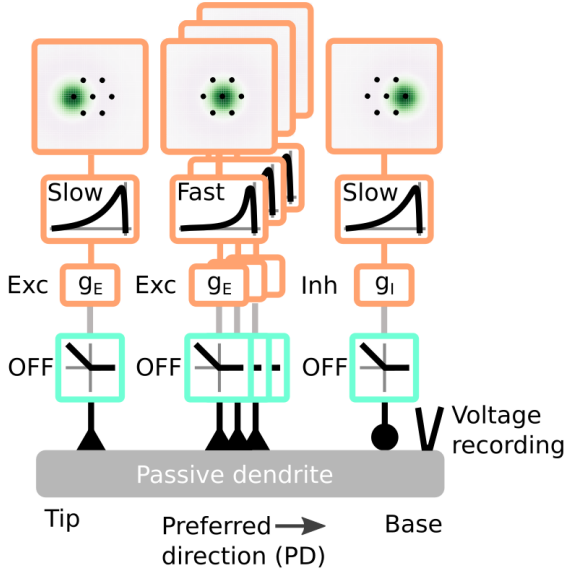

$\mathbf{f}$

Models of dendritic responses of T5 neurons

All rectified

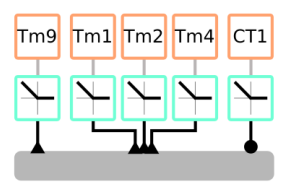

Tm9 linear

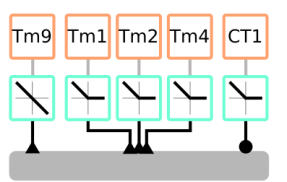

All linear

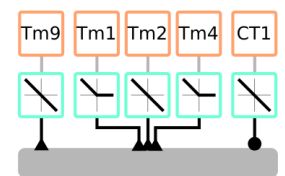

g Voltage responses of the T5 dendrite models

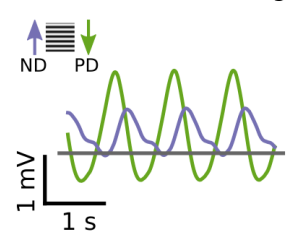

DSI: 0.28

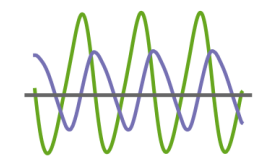

DSI: 0.18

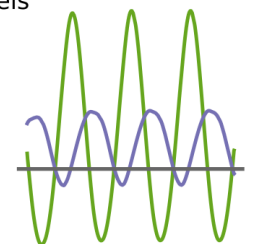

DSI: 0.33

Voltage responses of a T5 axon terminal

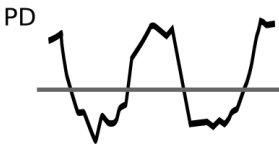

ND
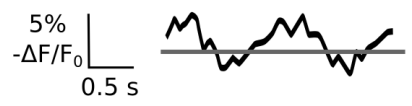

Figure 1: Circuit organization and models of local motion detection in Drosophila. Continued on next page. 
Figure 1: Circuit organization and models of local motion detection in Drosophila. (a) Schematic showing the dendrites of $\mathrm{T}_{4}$ neurons in the $\mathrm{ON}$ motion pathway. Inputs are organized from tip to base, corresponding to the trailing and leading site of the neuron's receptive field with respect to its preferred direction (PD): Mi9 (trailing, 1), Mi1 and Tm3 (central, 2), and Mi4 (leading, 3). Boxes show visual response properties. (b) Schematic showing the dendrites of $\mathrm{T}_{5}$ neurons in the OFF motion pathway. Inputs are organized from tip to base, matched to the visual sites in A as Tm9, Tm1/Tm2/Tm4, and CT1. (c) Schematic showing the receptive field organization of $\mathrm{T}_{5}$ neurons following Wienecke et al. (2018). (d) A linearnonlinear model simulates the responses of $\mathrm{T}_{5}$ input neurons (linear, orange; nonlinear, light green). A moving sine wave grating is processed by a hexagonal array of linear spatial (dark green spot) and temporal filters (black line), a conductance function $g$ and an output nonlinearity. The array matches the arrangement of units in the fly eye (red). (e) A full model of a $\mathrm{T}_{5}$ dendrite preferring downward motion. The input organization and properties follow those shown in (b) using the model components described in (d). All inputs are integrated by a passive dendrite, and the voltage response is recorded at its base. (f) Schematic showing $\mathrm{T}_{5}$ models: a model with all inputs OFF rectified, a model with a linear OFF input on the Tm9 site, and a model with all input sites receiving one linear OFF input. (g) Voltage responses in the preferred and null directions (ND) to a sinewave grating from the models in (f) (top), and from Wienecke at al. 2018 (bottom). Model responses are to a grating with a spatial frequency of $24 \mathrm{deg} / \mathrm{cycle}$ and temporal frequency of $1 \mathrm{~Hz}$. The direction-selectivity index (DSI) is shown below the traces, calculated from the amplitude of the Fourier component at $1 \mathrm{~Hz}$ of the voltage responses for each of the 16 directions of motion simulated (Fig. $\left.\mathrm{S}_{1} \mathbf{b}\right)$.

\section{Results}

\section{The presence of both $\mathrm{ON}$ and $\mathrm{OFF}$ inputs improves responses to motion}

A recent study showed that direction selectivity in the $\mathrm{T}_{4} \mathrm{ON}$ pathway can be modeled by combining three inputs providing slow OFF inhibition, fast ON excitation, and slow ON inhibition. ${ }^{37}$ In the OFF pathway, an analogous model would require an $\mathrm{ON}$ inhibitory input on the trailing site of the receptive field corresponding to the dendrite base, where $\mathrm{T}_{5}$ receives most synaptic inputs from Tm9 (Fig. 1a,b).

To test whether direction selectivity in $\mathrm{T}_{5}$ would indeed benefit from yet uncharacterized ON inputs, we modeled responses to sine gratings moving in different directions by simulating the $\mathrm{T}_{5}$ dendrite as a passive cable that integrates inputs from the main presynaptic neuron types. At each of the three input sites on the hexagonal ommatidia grid, a neuron's receptive field was represented with a linear-nonlinear (LN) model consisting of linear spatial and temporal filters, ${ }^{32}$ a synaptic function that transformed the filter response into a conductance change, an output nonlinearity, and an offset applied to prevent negative conductances (Fig. 1 d). The output of the LN model was fed into the corresponding site in the passive dendrite for each neuron type, weighted proportionally to their synaptic connectivity, ${ }^{13}$ the conductance inputs were transformed to input currents that were linearly added and integrated to obtain the membrane voltage. ${ }^{20,21,37}$ Finally, the output voltage response was recorded from the base of the $\mathrm{T}_{5}$ model dendrite (Fig. $\left.1 \mathbf{e}\right)$.

We considered several models differing in the response properties of the $\mathrm{T}_{5}$ inputs and compared 
them on the basis of their direction selectivity computed across 16 motion directions (Fig. $\mathrm{S}_{1}$ ), and show their response traces in the preferred and null directions. A model that received only OFF-rectified inputs produced direction-selective responses reproducing a mechanism consistent with the one described in, ${ }^{21}$ which requires OFF excitation over the trailing and central sites (here Tm9, Tm1, Tm2, and $\mathrm{Tm}_{4}$ ), and OFF inhibition in a smaller region on the leading site (here $\mathrm{CT}_{1}$ ). However, these responses failed to reproduce the sinusoidal responses of $\mathrm{T}_{5}$ neurons consisting of depolarizations to OFF and hyperpolarizations to ON grating components (Fig. 1 e,g). ${ }^{7}$ Because of Tm9's position on the trailing site of the $\mathrm{T}_{5}$ dendrite (Fig. $1 \mathbf{1}$ ), we next provided $\mathrm{T}_{5}$ with a linear input by using a linear output function for the excitatory Tm9 input. In this way, we kept the known OFF selectivity of Tm9 while still adding an hyperpolarizing ON component to the trailing site (Fig. 1 f). The linear Tm9 model responses had both ON hyperpolarizations and OFF depolarizations similar to biological $\mathrm{T}_{5}$ responses, but this model's direction selectivity was lower than the previous one (Fig. $1 \mathbf{f}, \mathbf{g})$, likely because it lacked ON inputs in the central and leading sites implementing the contrast-opponent properties of a $\mathrm{T}_{5}$ receptive field. A model with an additional linear output function for $\mathrm{Tm} 2$ in the central site produced qualitatively similar voltage responses, but improved direction selectivity for calcium responses (Fig. $\left.S_{1} \mathbf{a}, \mathbf{b}\right)$.

To address the potential benefit of $\mathrm{ON}$ inputs across the receptive field, we set additional linear output functions for $\mathrm{Tm}_{2}$ in the central site and CT1 in the leading site (Fig. 1 f). This model produced responses that were both direction selective and biologically plausible in that they had a sinusoidal shape with both hyperpolarizations to ON and depolarizations to OFF (Fig. $\mathbb{1}$ f,g). The previous conclusions hold across a range of parameters and stimulus conditions (Fig. $\mathrm{S}_{1}$ ). Thus, even a situation in which just one of the inputs is linear is already sufficient to produce $\mathrm{T}_{5}$ responses with ON and OFF components, but only one linear input at each dendrite site produces qualitatively similar responses with higher direction selectivity. This suggests that additional ON information from $\mathrm{T}_{5}$ presynaptic neurons might be present.

\section{Tm9 provides $\mathrm{ON}$ information required by $\mathrm{T}_{5}$}

Tm9 has been shown to have a central role in motion computation. It provides most synaptic input to $\mathrm{T}_{5}$, constitutes the sole input on the trailing site of the $\mathrm{T}_{5}$ receptive field and provides major input to CT1 on the leading site, and it leads to the strongest phenotypes when silenced alone or in combination with other Tm neurons. ${ }^{13,14,34}$ However, Tm9 neurons have been previously regarded as OFF rectified. ${ }^{32,34}$ To understand the origin of the ON input motivated by models of motion computation $^{7}$ (Fig. 1), we first characterized Tm9 receptive field properties to both ON and OFF stimuli using in vivo two-photon calcium imaging. We expressed the genetically encoded calcium indicator GCaMP6f in Tm9 neurons, and recorded calcium signals in response to visual stimuli from Tmy axon terminals in the first layer of the lobula (Fig. 2a).

To probe Tm9 spatial receptive fields we used ON bars (bright bars on a dark background) and OFF bars (dark bars on a bright background). Individual five-degree bars were shown as $0.5 \mathrm{~Hz}$ flicker. Tm9 neurons responded positively to OFF bars within a constrained region on the screen covering 6.9 degrees (Fig. 2b,c; Fig. S2b,c). Interestingly, Tm9 neurons responded with a decrease in calcium to ON bars (Fig. 2b). Individual Tm9 axon terminals responded negatively to ON bars covering a wider spatial range and sometimes spanning the whole stimulation area (Fig. $2 \mathbf{b}$; Fig. 
$\left.\mathrm{S}_{2} \mathbf{c}, \mathbf{d}\right)$. Quantification of the spatial receptive field showed that Tm9 ON responses were variable, ranging from $8.3 \mathrm{deg}$ to $71.5 \mathrm{deg}$ (mean $28.1 \mathrm{deg}$, std $17.6 \mathrm{deg}$, grouping both orientations). Negative Tm9 responses to $\mathrm{ON}$ bars (ON receptive field) were generally wider than positive Tm9 responses to OFF bars (OFF receptive field), both for horizontally and vertically oriented bars (Fig. $2 \mathbf{b , c})$. In summary, Tm9 neurons exhibit both an ON and an OFF receptive field component that differ in their spatial extent.

This larger ON receptive field size could account for a discrepancy in previous studies that reported narrow receptive fields observed with OFF bars, ${ }^{34}$ and wide receptive fields measured with white noise ${ }^{14}$ which could be explained by the differential activation of distinct ON and OFF receptive fields. White noise, containing both bright and dark bars, may activate the wide ON component of the receptive fields. In a new experimental set, we recorded Tm9 receptive fields for the same neurons using ON and OFF bars as well as ternary white noise (white, grey, or black stripes). To compare spatial receptive field properties across stimuli, we represented the two-dimensional receptive field as a sign-consistent Cartesian product of the one-dimensional receptive fields obtained from horizontal and vertical stimulus presentations (Fig. 2d). The receptive fields measured with ternary white noise were larger than the OFF but smaller than the ON receptive fields, seen in examples from six different flies, as well as in the population average (Fig. $2 \mathbf{e , f}$ ). Thus, stimuli that contain ON components elicit wider receptive fields than pure OFF stimuli, and there is some interaction between the two.

To investigate whether the measured receptive field properties were specific to Tm9, we first simultaneously measured the activity of Tm9 and another Tm neuron, Tm4. To record the activity of two neurons responding to the same point in visual space, we used different binary expression systems to express GCaMP6f specifically in Tm9 neurons and the red-shifted calcium indicator jRGECO1a in $\mathrm{Tm}_{4}$ (Fig. 3a). We further picked $\mathrm{Tm}_{4}$ because of its spatial separation from Tm9 terminals. ${ }^{12}$ Tm9 and $\mathrm{Tm}_{4}$ both responded to full field OFF flashes with sustained and transient kinetics. However, Tm9 responded stronger to the grey-OFF transition, consistent with Tm9 receiving input from the luminance-sensitive $\mathrm{L}_{3}$ neuron. ${ }^{38} \mathrm{Tm}_{4}$ did not respond to $\mathrm{ON}$ flashes, whereas Tm9 showed weak decreases in calcium signal in response to full field ON flashes (Fig. 3 b). This difference became more prominent when extracting receptive fields from local bar stimuli. No responses to bright bars were detectable in Tm4 neurons, whereas Tm9 responded negatively to narrow bright bars, and displayed a wide $\mathrm{ON}$ receptive field (Fig. 3c-e; $\mathrm{S}_{3}$ ). This was visible in individual ROI responses (Fig. 3c) as well as in the population average (Fig. 3d). Spatial OFF receptive fields were narrow for both $\mathrm{Tm} 9$ and $\mathrm{Tm}_{4}$, consistent with previous reports ${ }^{34}$ (Fig. $3 \mathbf{c}$-e). Since Tm9 responses in the receptive field center reverse in sign upon reversing the stimulus polarity, one can think of them as having a receptive field structure resembling that of the LGN inputs to simple cells in mammalian $\mathrm{V}_{1}$. The existence of a negative $\mathrm{ON}$ receptive field suggests that $\mathrm{Tm}_{9}$ is a suitable candidate to provide the neural substrate for the $\mathrm{ON}_{5}$ input implementing a linear mechanism of direction selectivity on the trailing site of the $\mathrm{T}_{5}$ receptive field. 
a

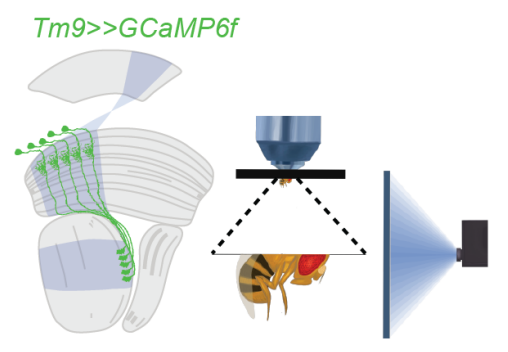

d

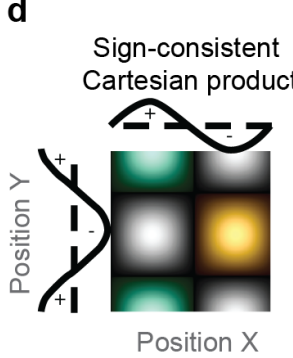

b

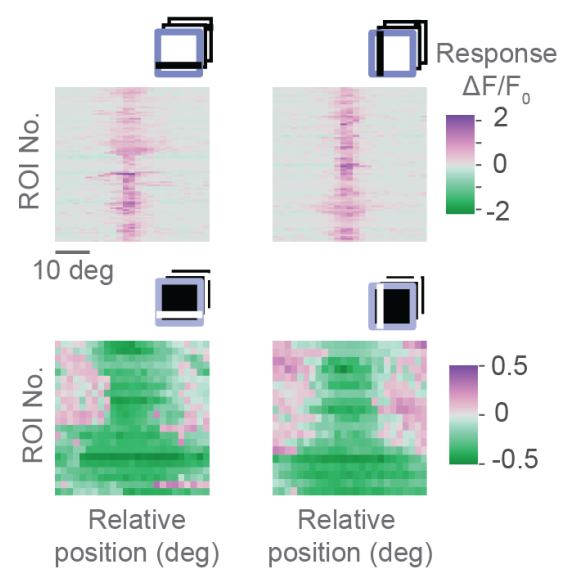

C

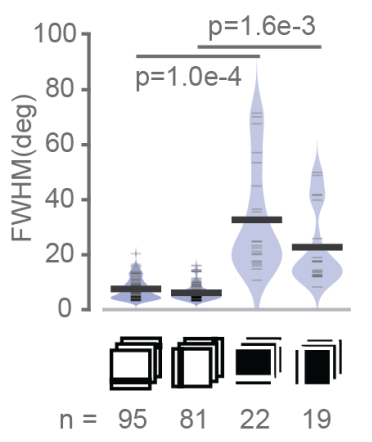

e

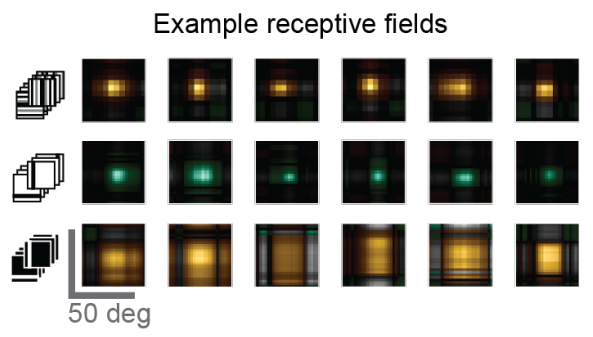

f

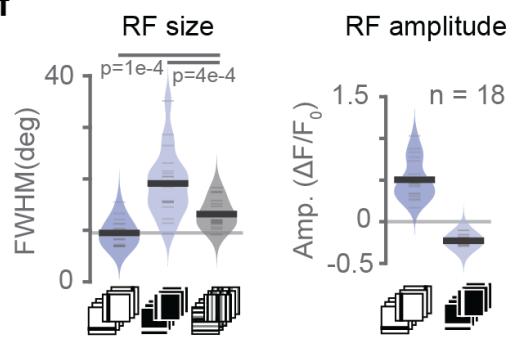

Figure 2: Tmg has wide ON and narrow OFF receptive fields. (a) Schematic showing the experimental setup used for in vivo two-photon calcium imaging of Tm9 neurons expressing GCaMP6f under visual stimulation. Visual information (blue) passes from the lamina (La) through the medulla (Me) housing Tm9 neurons into the lobula (Lo), where we recorded from Tm9 axon terminals (example image from a recording shown in the bottom). (b) Receptive fields obtained from Tm9 responses to ON and OFF bars in vertical and horizontal orientations. Receptive field centers are aligned for illustrative purposes. (c) Full width at half maximum (FWHM) of a single Gaussian fitted to the receptive fields in (b). (d) Two-dimensional visualization of spatial receptive fields by color-coding the Cartesian product of two orthogonal one-dimensional receptive fields (from calcium signal or filter amplitude) according to the sign and response strength: positive in both orientations in green, negative in both orientations in yellow, and opposing signs in gray. Color luminance linearly encodes the magnitude of the Cartesian product. (e) Receptive fields obtained with different stimuli for the same neurons: ternary white noise (top), OFF bars (middle), and ON bars (bottom). Panel shows six example neurons from six flies. (f) Amplitude and FWHM obtained from fitting a single Gaussian to each neuron's receptive field in the population $(n=18)$. In $(\mathbf{c})$ and $(\mathbf{f}) \mathrm{p}$-values come from two-tailed permutation tests. 
a

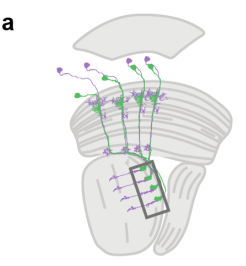

b

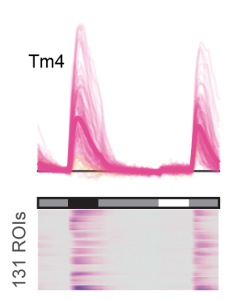

c

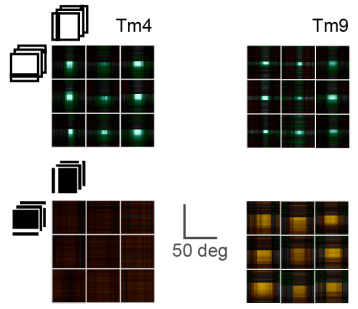

d
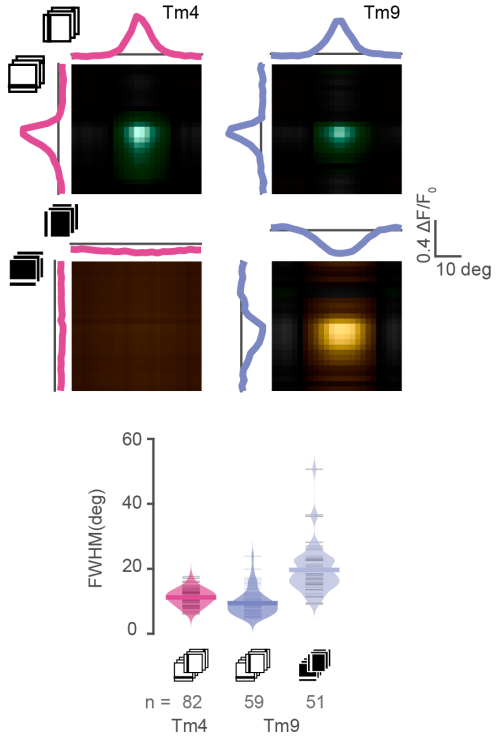

Figure 3: Dual-color imaging reveals specificity of the Tm9 ON receptive field. (a) Experimental setup used for in vivo two-photon calcium imaging under visual stimulation of Tm9 neurons expressing GCaMP6f (green), and Tm4 neurons expressing jRGECO1a (purple). (b) Responses to full-field ON and OFF flashes from an intermediate gray background. Both $\mathrm{Tm} 9$ (blue) and $\mathrm{Tm}_{4}$ (pink) respond preferentially to OFF transitions, but only Tm9 responds (negatively) to ON flashes. (c) Receptive fields of nine example neurons from each neuron type recorded simultaneously. Only Tm9 has ON receptive fields. (d) Population average of receptive fields simultaneously recorded and aligned to the center for visualization by maximizing their cross-correlation. Both Tm9 and $\mathrm{Tm}_{4}$ have narrow OFF receptive fields but only Tm9 has a wide ON receptive field. (e) Full width at half maximum (FWHM) obtained from fitting a single Gaussian to each neuron's receptive field in the population. Tm9 ON (light blue, $n=51$ ), Tm9 OFF (blue, $n=59$ ), and Tm4 OFF (pink, $\mathrm{n}=82$ ). $\mathrm{Tm}_{4}$ did not have an $\mathrm{ON}$ receptive field. Values are averages over the two orientations. 


\section{Tm2 and CT1 provide additional, central and trailing $\mathrm{ON}$ inputs to $\mathrm{T}_{5}$}

In addition to the Tm9 ON receptive field, $\mathrm{ON}$ inputs at the central and leading sites can explain the proposed linear mechanism of direction selectivity ${ }^{7}$ (Fig. 1). On the central site, $\mathrm{T}_{5}$ gets most of the inputs from $\mathrm{Tm} 1, \mathrm{Tm} 2$, and Tm $4 .{ }^{13}$ To test whether any of those could provide a central ON input to $\mathrm{T}_{5}$, we characterized their $\mathrm{ON}$ receptive field properties using narrow bars. For a comprehensive analysis of all Tm neurons, we expressed GCaMP6f in Tm1, Tm2, Tm4, or Tm9, and recorded calcium signals in response to ON and OFF bars from the axon terminals of each neuron type (Fig. ${ }_{4} \mathbf{a}-\mathbf{h}, \mathrm{S}_{4}$ ). All Tm neurons responded positively to OFF bars within a constrained region

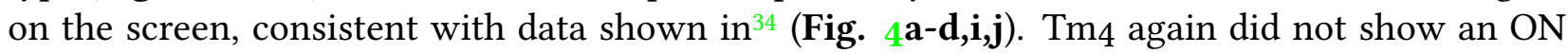
receptive field, this time imaged with GCaMP6f instead of jRGECO1a, showing that this is not due to the indicator used. Tm1 also did not respond to ON bars. Interestingly, both Tm9 neurons and Tm2 neurons responded negatively to ON bars with a mean full width at half maximum (FWHM) of $13 \mathrm{deg}(\mathrm{Tm} 2)$, and $17 \mathrm{deg}(\mathrm{Tm} 9)$ (Fig. $4 \mathbf{f}, \mathbf{h}, \mathbf{k}, \mathbf{l})$. Both ON and OFF bar responses were similar for horizontally and vertically oriented bars. Therefore, Tm2 has both an ON and an OFF receptive field component that differ in their spatial extent. Altogether, our results show that Tm2 can provide the ON input to the central site of the $\mathrm{T}_{5}$ receptive field required by recent data demonstrating linear summation, ${ }^{7}$ and that additionally improves direction-selectivity in nonlinear models (Fig. 1 ).

The $\mathrm{T}_{5}$ leading site gets most of its inputs from $\mathrm{CT}_{1}$ neurons, which in turn receive most of their inputs from Tm9. ${ }^{13}$ Thus, the ON input from Tm9 can in principle be conveyed to the leading site through CT1. Furthermore, $\mathrm{CT}_{1}$ is thought to provide GABAergic inhibition to $\mathrm{T}_{5} \cdot{ }^{11}$ To test what spatial contrast information $\mathrm{CT}_{1}$ provides to $\mathrm{T}_{5}$, we measured responses to $\mathrm{ON}$ and $\mathrm{OFF}$ bars from axon terminals in the lobula layer 1 of CT1 neurons expressing GCaMP6f (Fig. $5 \mathbf{a})$. CT1 showed positive, spatially confined responses to OFF bars with a FWHM of approximately 5 deg (Fig. $5 \mathbf{b}$ ), consistent with previous studies. ${ }^{36}$ Moreover, $\mathrm{CT} 1$ responded positively to ON bars in the locations surrounding the OFF receptive fields, and decreased responses in the location where it was excited by OFF bars (Fig. $5 \mathbf{c}, \mathbf{d}$ ). Because $\mathrm{CT}_{1}$ responds positively to OFF stimuli and negatively to ON, it can not only provide $\mathrm{T}_{5}$ with the asymmetric OFF inhibition supported by nonlinear models, ${ }^{21}$ but also the complementary, dishinibitory ON input supported by linear, contrast-opponent models (Fig. ${ }_{1}$ b). ${ }^{7}$ Therefore, $\mathrm{T}_{5}$ can access $\mathrm{ON}$ information throughout its receptive field.

\section{Tm9 variability correlates with $T_{5}$ tuning properties}

We next asked whether Tm9 properties shape downstream $\mathrm{T}_{5}$ computations. To do this, we harnessed the variability observed in Tm9 responses (Fig. 2c), and asked whether this is passed on to downstream computations. We focused on this neuron again, because blocking Tm9 had the strongest effect on direction-selective responses downstream, ${ }^{14,34}$ and because of Tm9 being T5's most numerous input. We simultaneously recorded responses from Tm9 axon terminals and downstream $\mathrm{T}_{5}$ dendrites and asked whether $\mathrm{Tm}_{9}$ variability is reflected in the properties of downstream $\mathrm{T}_{5}$ neurons. To do so, we expressed jRGECO 1 a in Tm9 and GCaMP6f in $\mathrm{T}_{5}$ and recorded signals in the lobula plate, where $\mathrm{Tm}_{9}$ axon terminals synapse onto $\mathrm{T}_{5}$ dendrites (Fig. 6 a). Since $T_{5}$ neurons exist in four subtypes, each selective for one of the four cardinal directions of motion, each $\mathrm{Tm} 9$ axon terminal contacts the dendrites of four different $\mathrm{T}_{5}$ neurons. By virtue 
a
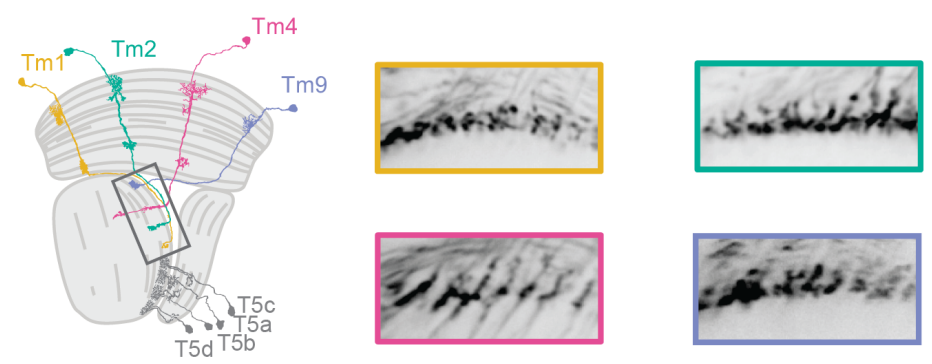

b

C

$\mathrm{Tm} 2$

d
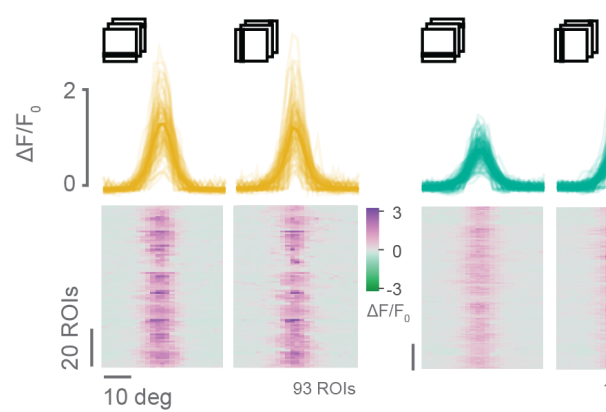

f

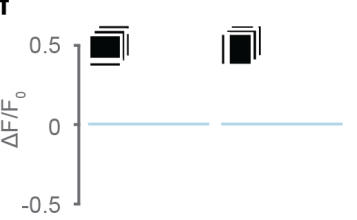

g

h

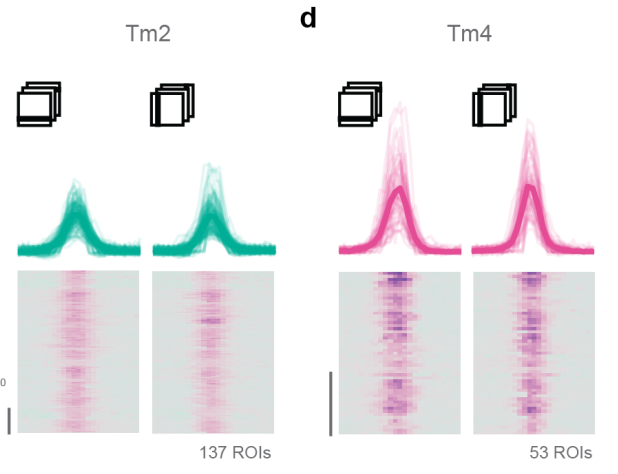

e $\quad \operatorname{Tm} 9$
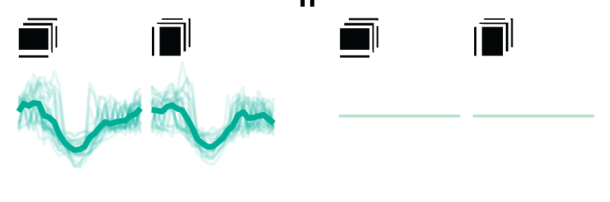

i
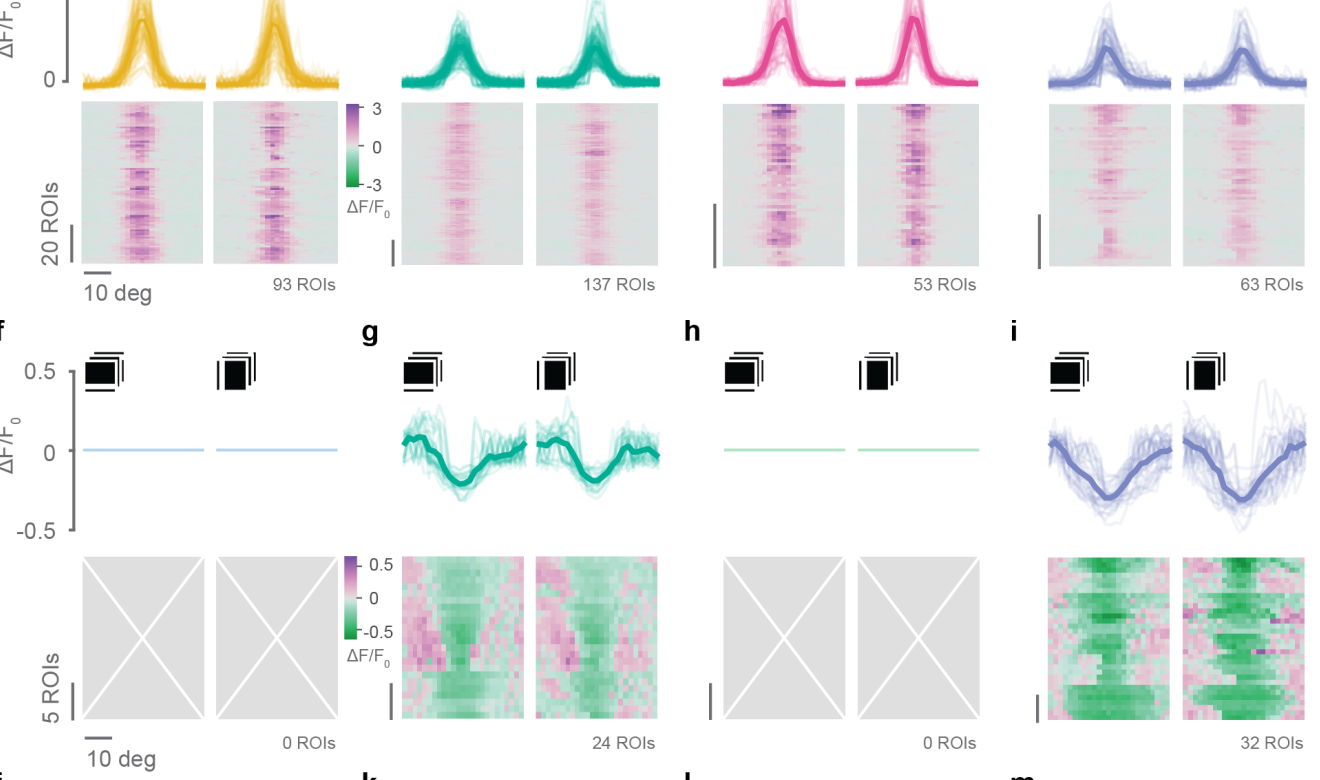

j
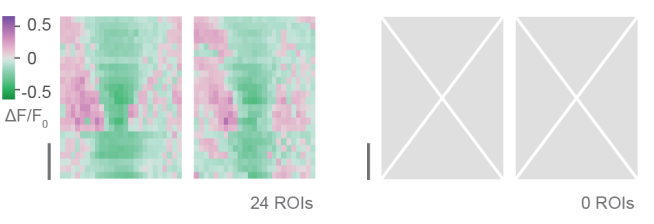

$\mathbf{k}$
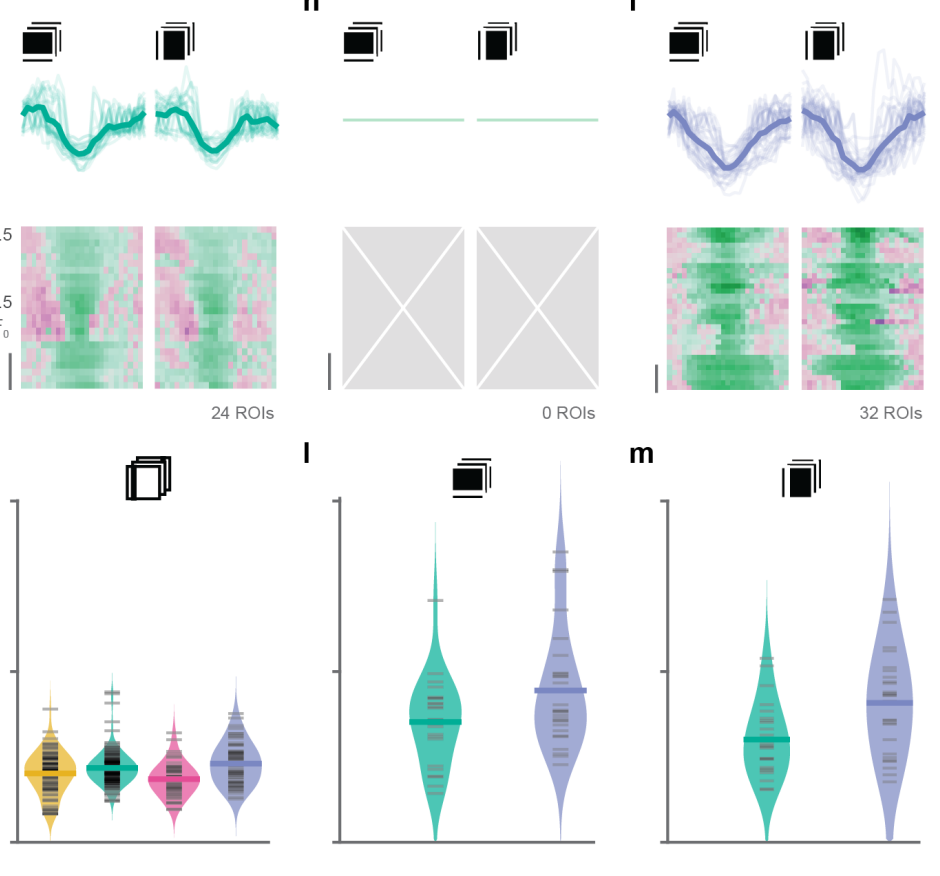

m

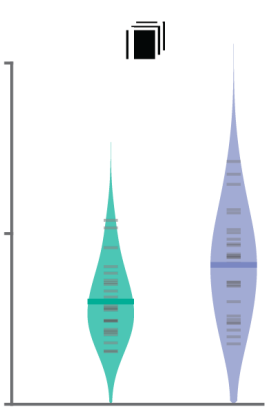

Figure 4: Tm2 and Tm9 neurons have ON receptive fields. (a) Schematic of the optic lobe showing the anatomy of the main medulla input neurons to $\mathrm{T}_{5}, \mathrm{Tm}_{1}, \mathrm{Tm}_{2}, \mathrm{Tm}_{4}$, and $\mathrm{Tm} 9$, and example images from recordings of their axon terminals.(b-e) OFF receptive fields obtained from in vivo two-photon calcium imaging of responses to vertical and horizontal dark bars from $\operatorname{Tm} 1$ (b), $\operatorname{Tm} 2$ (c), $\operatorname{Tm}_{4}$ (d), and Tm9 (e) neurons expressing GCaMP6f. Only neurons with responses in both orientations fitted by a single Gaussian with $r^{2}>0.2$ were included. Number of ROIs (neurons) is shown below the plots on the right. (f-i) ON receptive fields obtained from responses to vertical and horizontal bright bars from $\operatorname{Tm} 1$ (f), $\operatorname{Tm} 2(\mathrm{~g}), \operatorname{Tm}_{4}$ (h), or Tm9 (i) neurons in (b-e). Only Tm2 and Tm9 neurons had ON receptive fields. (j-m) Full width at half maximum (FWHM) from receptive fields obtained from horizontal OFF bars (j), vertical OFF bars $(\mathbf{k})$, horizontal ON bars (l), and vertical bars $(\mathbf{m})$. Data points correspond to neurons in (b-i) matching the stimulus condition. Sample sizes are indicated below the panels. 
a

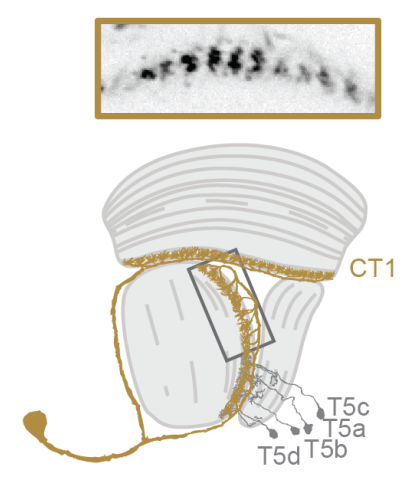

d

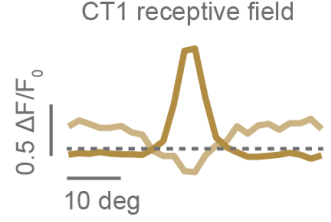

b
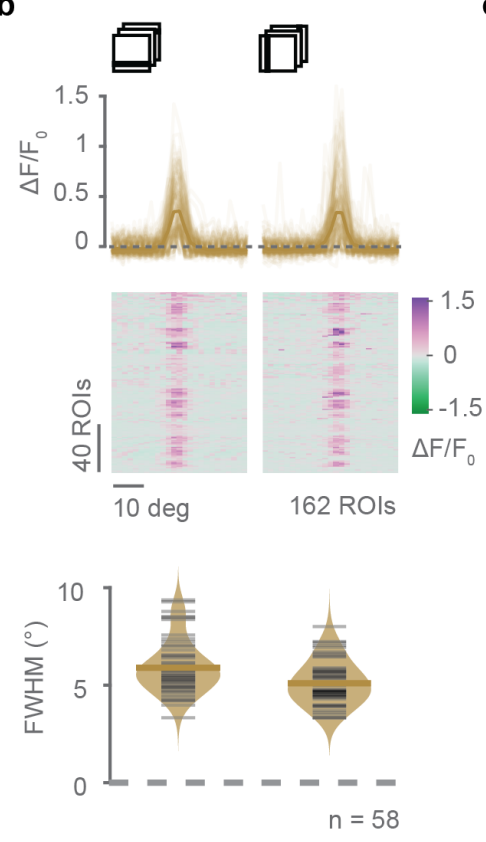

C

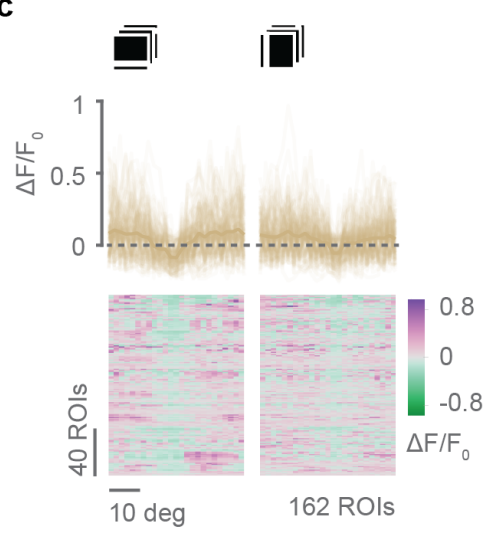

NA

NA

$n=0$

Figure 5: CT1 neuron responses contain $\mathrm{ON}$ and $\mathrm{OFF}$ information. (a) Example average intensity projection from recordings of axon terminals from a single CT1 neuron (top), and a schematic of the optic lobe showing the anatomy of the CT1 neuron (bottom). (b) Top: OFF receptive fields obtained from in vivo two-photon calcium imaging of responses to vertical and horizontal dark bars from CT1 neurons expressing GCaMP6f. Showing all recorded ROIs $(n=162)$. Bottom: Full width at half maximum (FWHM) from receptive fields obtained from horizontal, and vertical OFF bars from ROIs in top panel fitted by a Gaussian. Only neurons (ROIs) with response quality index above 0.5 and $r^{2}>0.2$ are shown, similarly to Fig. $4 \mathbf{j}-\mathbf{m}$. (c) As in (b) but for ON receptive fields. Top: CT1 responses to vertical and horizontal ON bars. Bottom: The CT1 ON receptive fields could not be well-fitted by a Gaussian. (d) Average receptive fields from vertical OFF and ON bars from $(\mathbf{b}, \mathbf{c})$ overlaid to visualize the spatial organization of CT1 responses to ON and OFF bars. 
of $\mathrm{T}_{5}$ orientation selectivity, ${ }^{8}$ horizontal stimuli predominantly stimulate the two $\mathrm{T}_{5}$ subtypes selective for upward and downward motions, and vertical stimuli predominantly excite leftward and rightward selective $\mathrm{T}_{5}$ subtypes.

We first recorded receptive fields using $\mathrm{ON}$ and OFF bars. Both $\mathrm{Tm} 9$ and $\mathrm{T}_{5}$ responded positively to OFF bars, whereas only Tm9 showed prominent negative responses to ON bars, demonstrating that we can spectrally separate $\mathrm{Tm}_{9}$ and $\mathrm{T}_{5}$ signals (Fig. $6 \mathbf{b}-\mathbf{e}$ ). We extracted the OFF receptive field properties from single Gaussian fits to the tuning curves. A tight correlation for the receptive field position confirmed that $\mathrm{Tm}_{9}$ and $\mathrm{T}_{5}$ neurons that were recorded together were responding to the same point in visual space (Fig. $\left.\mathrm{S}_{5} \mathbf{a}, \mathbf{b}\right)$. Interestingly, the widths of $\mathrm{T}_{5}$ and $\mathrm{Tm}_{9}$ OFF receptive field were positively correlated, indicating that a larger Tm9 receptive field generates a larger $\mathrm{T}_{5}$ receptive field (Fig. 6f). This suggests that the variability observed in receptive fields is passed on to downstream computation and confirms tight coupling between $\mathrm{Tm}_{9}$ and $\mathrm{T}_{5}$ properties. To test whether this functional correlation extends to moving stimuli that include both ON and OFF components, we next measured $\mathrm{Tm}_{9}$ and $\mathrm{T}_{5}$ responses to moving sine wave gratings. We measured the spatiotemporal frequency tuning of these neurons using gratings of different spatial and temporal frequencies. Gratings were moving leftward to selectively stimulate one subtype of $\mathrm{T}_{5}$ selective for that motion direction. We quantified the spatiotemporal frequency tuning of a neuron using the response amplitude of the Fourier component matching the temporal frequency of the grating. Average tuning properties across the population of all recorded $\mathrm{Tm}_{9}$ and $\mathrm{T}_{5}$ cells were highly similar both spatially and temporally (Fig. 6g). Comparing the tuning between individual $\mathrm{Tm}_{9}$ and $\mathrm{T}_{5}$ neuron pairs showed a strong pairwise correlation with a mean correlation coefficient of 0.8 (Fig. $6 \mathbf{h}$ ). Overall, Tm9 and $\mathrm{T}_{5}$ response properties are correlated for static stimuli, as well as for moving stimuli with both $\mathrm{ON}$ and OFF components. This functional link further supports Tm9's important role in the computation of direction selectivity by $\mathrm{T}_{5}$, and the biological relevance of variability mediated through Tm9. 
bioRxiv preprint doi: https://doi.org/10.1101/2021.04.17.440268; this version posted April 19, 2021. The copyright holder for this preprint (which was not certified by peer review) is the author/funder, who has granted bioRxiv a license to display the preprint in perpetuity. It is made available under ACC-BY-NC-ND 4.0 International license.

a

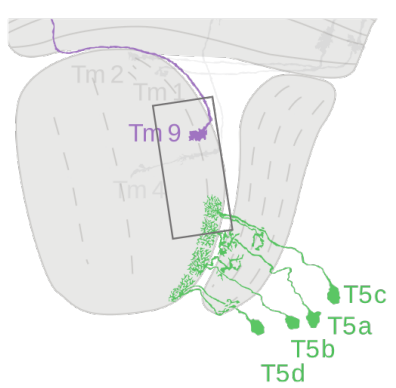

b

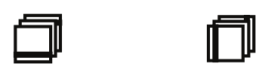

T5 receptive fields
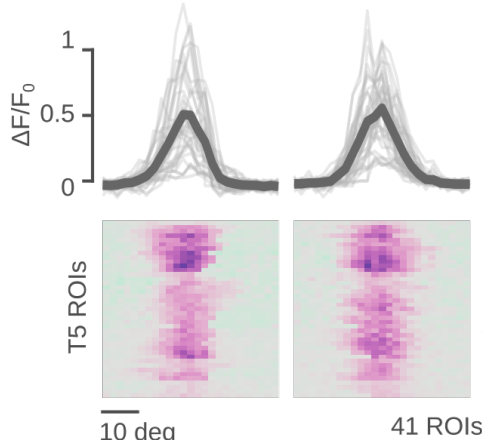

d

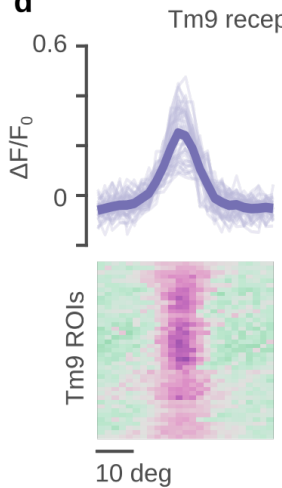

f
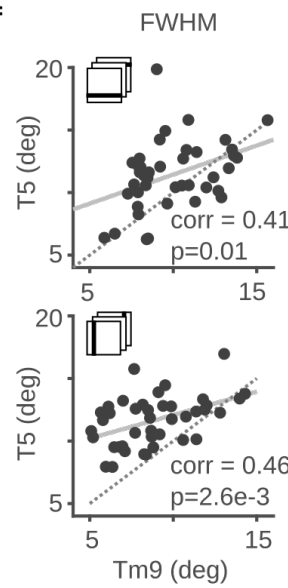

41 ROls

g
T5-GCaMP6f

Tm9-jRGECO1a

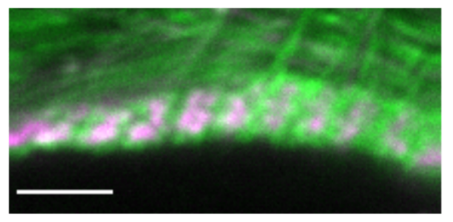

C

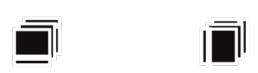

T5 receptive fields
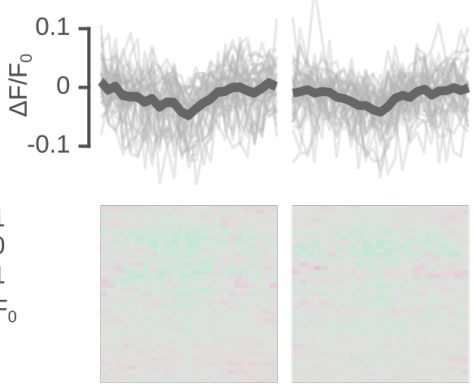

e

e Tm9 receptive fields
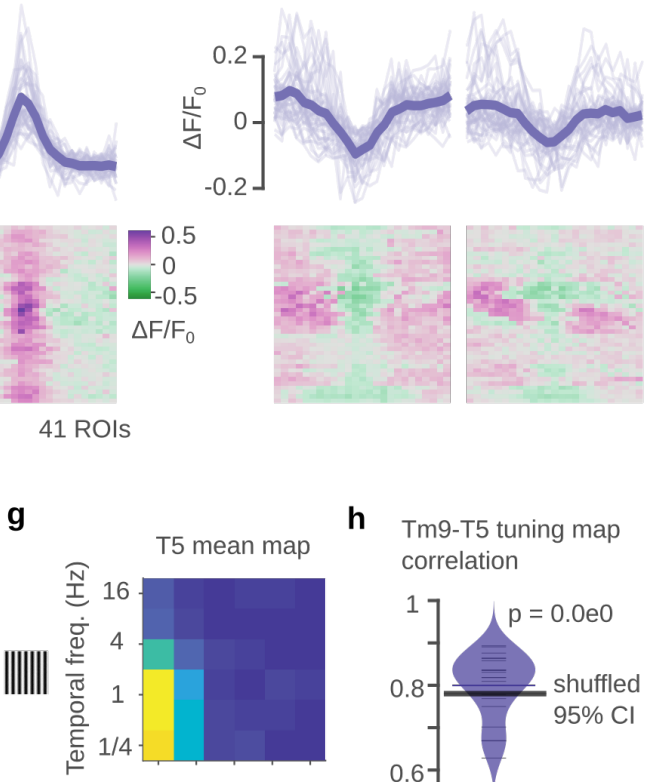

Tm9 mean map

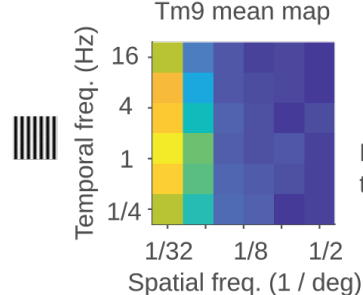

h

Tm9-T5 tuning map

correlation
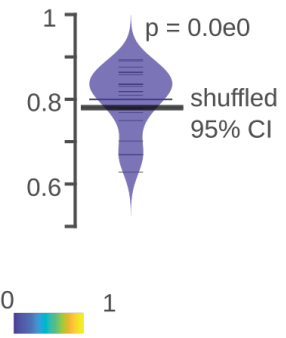

Normalized

tuning (a.u.)

Figure 6: Tm9 variability correlates with $\mathbf{T}_{5}$ functional properties. Continued on next page. 
Figure 6: $\mathbf{T m} 9$ variability correlates with $\mathbf{T}_{5}$ functional properties. (a) Schematic showing the fly optic lobe. Inset shows an example image from a recording of $\mathrm{T}_{5}$ neurons expressing GCaMP6f (green) and Tm9 neurons expressing jRGECO1a (purple). (b,c) OFF (b) and ON (c) receptive fields obtained from in vivo two-photon calcium imaging from responses to vertical and horizontal dark bars from $\mathrm{T}_{5}$ neurons expressing GCaMP6f. Same neurons for ON and OFF $(\mathrm{n}=41)$. T5 did not respond to ON bars. (d,e) OFF (d) and ON (e) receptive fields Tm9 neurons expressing jRGECO1a imaged simultaneously and overlapping with the $\mathrm{T}_{5}$ dendrites in (b,c). (f) Full width at half maximum (FWHM) for OFF receptive fields of Tm9 vs $\mathrm{T}_{5}$ signals for horizontal (top) and vertical bars (bottom). Tm9 and $\mathrm{T}_{5}$ signals originate from the same region of interest. Pearson's correlations $=\operatorname{corr}(n=41)$. Solid line is the least-squares fit. $T_{5}$ signals had greater FWHM than Tm9 signals, as seen by the points lying above the identity line (dotted). (g) Average spatiotemporal tuning maps for $\mathrm{T}_{5}$ (top) and $\mathrm{Tm}_{9}$ (bottom). Maps were obtained from the Fourier amplitude component matching the temporal frequency of the sinewave gratings of different spatial and temporal frequencies moving leftward. Maps were scaled by their maximum response before averaging. Both Tm9 and $\mathrm{T}_{5}$ were selective for similar spatial and temporal frequencies. (h) Distribution of pairwise correlations between the spatiotemporal maps of $\mathrm{T}_{5}$ and $\mathrm{Tm} 9$ signals. Mean correlation is about o.8. To test whether pairwise correlations were sensitive to the particular pairs, we performed $1 \mathrm{e} 6$ random shuffles of the pairings of Tm9- $\mathrm{T}_{5}$ signals and quantified the $95 \%$ confidence interval of the mean correlation per shuffle. No random shuffle showed a larger mean correlation than the original mean correlation. Thus, he mean correlation is above the upper $95 \%$ confidence bound and confirms that $\mathrm{T}_{5}$ neurons are more correlated with their Tm9 input neurons than with other Tm9 neurons.

\section{Octopamine signaling sharpens the ON receptive field of Tm9}

The tuning of visual behaviors and of direction-selective cells depends on the behavioral state of the animal. ${ }^{32,39-41}$ Tm9 is required for direction-selective responses across a wide range of speeds. ${ }^{14,34}$ This suggests that Tm9 properties should be maintained across behavioral states, such as flying or walking, where the scene moves at faster speeds relative to the fly. Furthermore, to accurately compute local motion cues, it appears disadvantageous to sample across larger regions of visual space. Ideally, Tm9 neurons should display both negative ON and positive OFF responses across behavioral states, but these responses should be sharpened when the animal is walking or flying. To test this hypothesis, we recorded from Tm9 upon application of chlordimeform (CDM), an octopamine agonist that mimics active behavioral states and modulates the gain and selectivity of direction-selective cells..$^{32,39-43} \mathrm{Tm} 9$ responded negatively to $\mathrm{ON}$ and positively to OFF bar stimulus polarities with or without CDM (Fig. 7a,b). CDM appeared to decrease OFF center responses and strengthen the surround (Fig. $7 \mathbf{a}$ ). To quantify the center-surround interaction, we fitted a difference-of-Gaussians to the receptive fields. Indeed, CDM application significantly decreased the OFF receptive field center amplitude (Fig. 7c; Fig. S6a). Both center and surround amplitudes of the ON receptive fields reduced upon CDM application (Fig. $7 \mathbf{c}$ ), while absolute minimum and maximum responses did not change (Fig. S6a). Furthermore, the OFF receptive field surround and the ON receptive field center became significantly sharper in the presence of CDM (Fig. 7 d), resulting in sharpened net receptive fields (Fig. S6b), whereas the variability of Tm9 responses was not affected by CDM. Comparing center and surround in individual neurons showed that CDM did not change correlations of center-surround amplitudes for OFF bars and reduced them for ON bars 
(Fig. $7 \mathbf{e})$.

This center-surround organization in the presence of CDM suggests that responses to global OFF stimuli might be suppressed. When we measured Tm9 responses to full-field flashes, we indeed observed that Tm9 responses decreased to OFF flashes (Fig. $7 \mathbf{f}, \mathbf{g})$. Conversely, Tm9 negative responses to full-field $\mathrm{ON}$ stimuli were unchanged, again consistent with the net center-surround amplitude of ON receptive fields, that did not change in the presence of CDM (Fig. 7f,g; Fig. S6a). Taken together, our data show that octopamine signaling, mimicking an active behavioral state, sharpens the spatial receptive field of Tm9 neurons, while maintaining both negative ON and positive OFF components for motion estimation.?

\section{Discussion}

In this study, we sketched a minimal model that exposed the benefit of $\mathrm{ON}$ information to $\mathrm{T}_{5}$ and identified the $\mathrm{ON}$ inputs that provide the cellular basis to implement a mechanism for direction selectivity in the fly OFF motion pathway. Specifically, three OFF pathway interneurons, Tm9, Tm2, and $\mathrm{CT}_{1}$ have negative $\mathrm{ON}$ receptive field components, providing $\mathrm{ON}$ information to different spatial locations of the $\mathrm{T}_{5}$ spatial receptive field (Fig. $\left.7 \mathbf{h}, \mathbf{i}\right)$. Tm9 exhibits variability in its physiological receptive field properties, which are tightly coupled with downstream $\mathrm{T}_{5}$ properties. The newly identified ON properties of Tm9 are maintained in the presence of an octopamine receptor agonist, whereas spatial tuning of Tm9 is sharpened, matching constraints imposed by an active behavioral state.

\section{The neural basis for mechanisms of direction selectivity in the OFF path- way}

Our study provides critical insights into the physiological implementation of direction selectivity, and thus synthesizes different observations into a single mechanism: direction selectivity emerges from the integration of three spatial inputs with both ON and OFF responses. A minimal model in the ON pathway suggested that one input neuron with the opposite response polarity (here: OFF) on the trailing site of the $\mathrm{T}_{4}$ receptive field suffices to implement a passive mechanism of direction selectivity. ${ }^{20,37}$ Voltage recordings in $\mathrm{T}_{5}$ showed that passive integration also occurs in the OFF pathway. ${ }^{21}$ Another study that focused on $\mathrm{T}_{5}$ revealed that it has both $\mathrm{ON}$ and OFF subunits that are contrast opponent, i.e. reversing the stimulus polarity at each receptive field location leads to a reversal in response polarity. ${ }^{7}$ This contrast-opponent receptive field structure is used to produce direction selectivity through linear summation., ${ }^{717}$ Thus, receiving both ON and OFF information at all input locations agrees with the recently reported linearity of $\mathrm{T}_{5}$ receptive fields. Whether or not the same structure applies to $\mathrm{T}_{4}$ in the $\mathrm{ON}$ pathway remains to be seen. Interestingly, a recent model of illusory motion perception in Drosophila, required that the inhibitory input at the leading site of $\mathrm{T}_{4}\left(\mathrm{Mi}_{4}\right)$ responded linearly, to convey inhibition to $\mathrm{ON}$ and dishinibition to OFF. ${ }^{44}$ Analogously, the inhibitory input on the leading site of $\mathrm{T}_{5}, \mathrm{CT}_{1}$, provides inhibition to OFF and dishinibition to $\mathrm{ON}$ (Fig. 5). ${ }^{11}$ This suggests $\mathrm{T}_{4}$ and $\mathrm{T}_{5}$ at least share a similar input mechanism on their trailing sites. 


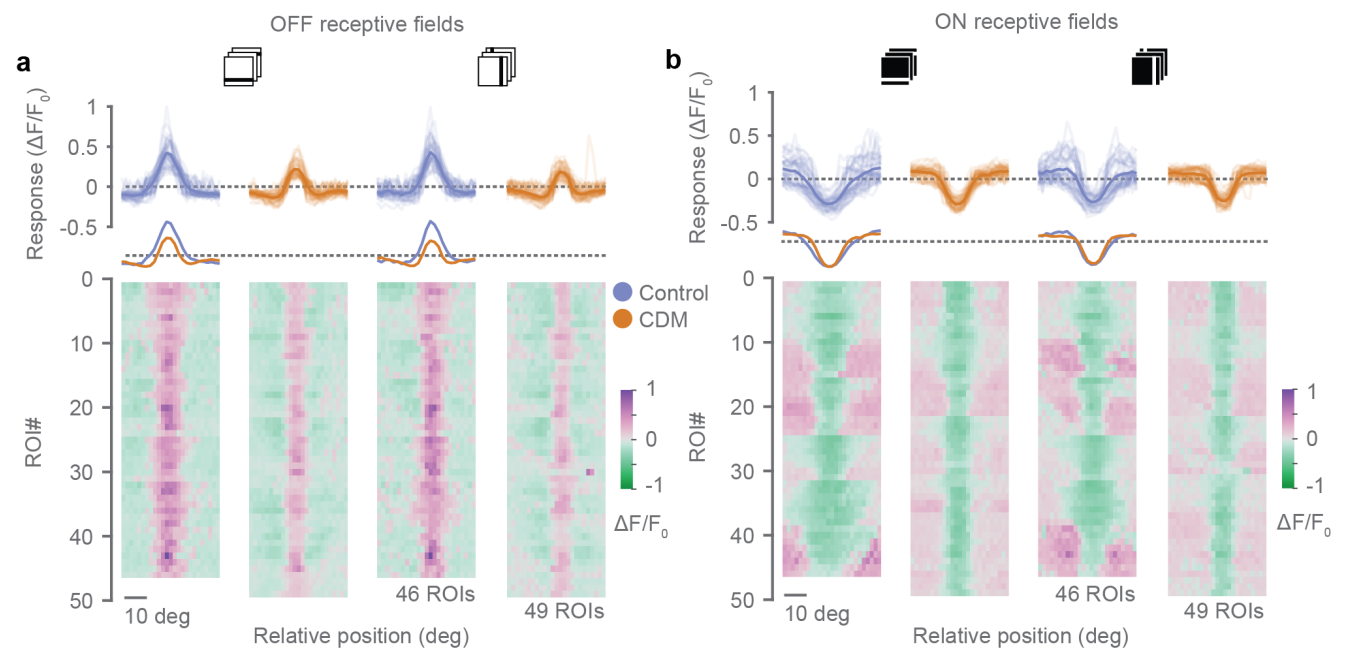

C $\begin{array}{cccc}\text { OFF } & \text { OFF } & \text { ON } & \text { ON } \\ \text { center } & \text { surround } & \text { center } & \text { surround }\end{array}$

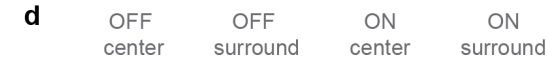
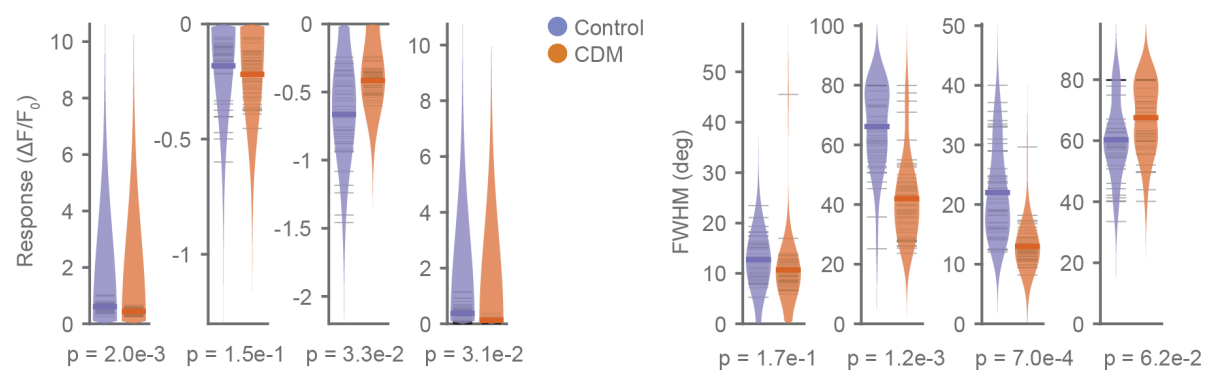

e
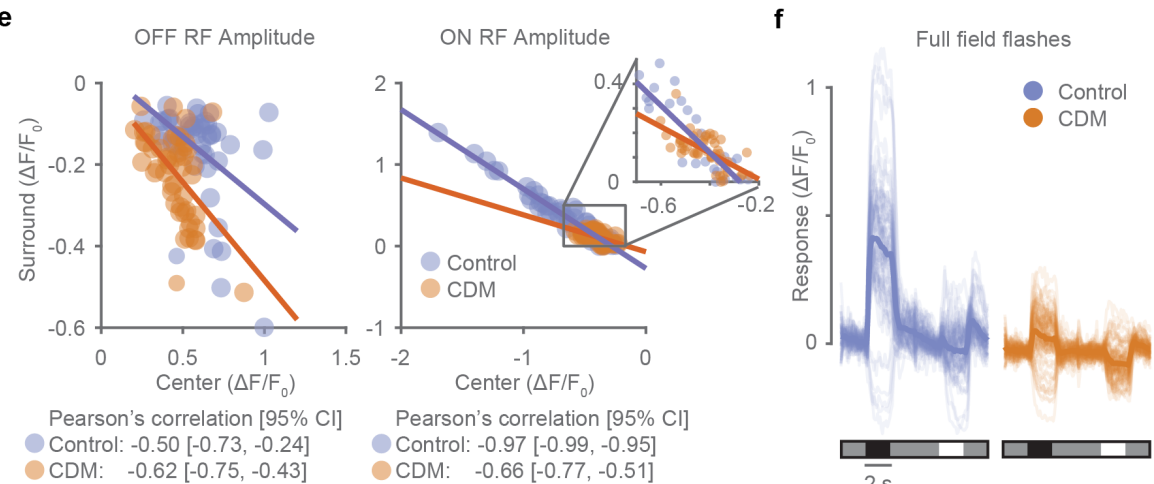

g

Pearson's correlation $[95 \% \mathrm{Cl}]$ Control: $-0.50[-0.73,-0.24]$ CDM: $\quad-0.62[-0.75,-0.43]$ CDM: $-0.66[-0.77,-0.51]$

h T5 voltage receptive field

i

Calcium responses of $\mathrm{T} 5$ input neurons

j
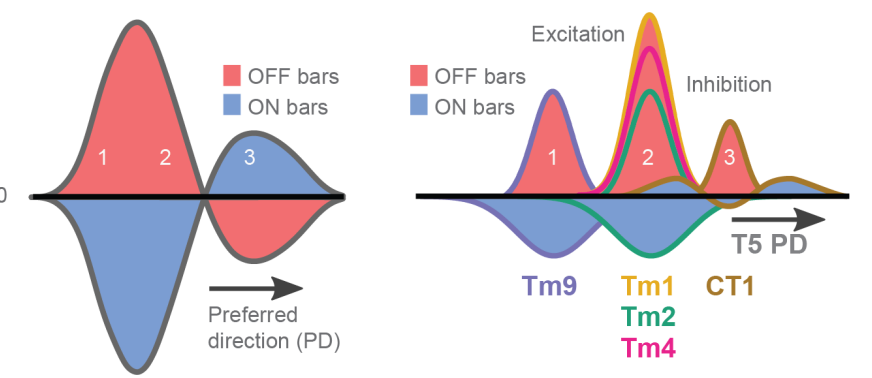

Input receptive fields under octopamine effects

- - Control

CDM (octopamine agonist)

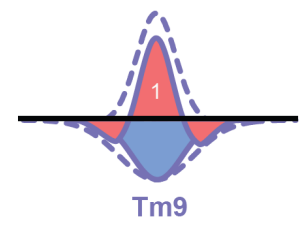

Figure 7: CDM sharpens Tm9 receptive fields. Continued on next page. 
Figure 7: CDM sharpens Tmg receptive fields. (a,b) OFF (a) and ON (b) receptive fields obtained from in vivo two-photon calcium imaging from responses to vertical and horizontal dark bars from Tm9 neurons expressing GCaMP6f, in the absence (blue) and in the presence of CDM (orange). Traces below controls are the mean curves scaled by the peak to display the surround increase in CDM in (a). Same neurons for $\mathrm{ON}$ and OFF: control $\mathrm{n}=46, \mathrm{CDM} n=49$. $(\mathbf{c}, \mathbf{d})$ Quantification of the receptive field center and surround amplitudes (c), and receptive field center full width at half maximum (FWHM) (d), obtained from a differenceof-Gaussians fit (data in a,b). Each point is the average of the fit amplitude (c) or fit FWHM (d) of a neuron across orientations. Two-tailed permutation tests comparing controls and CDM, p-values are indicated. (e) Comparison of receptive field amplitude of center and surround components for ON and OFF receptive fields shown in (c). Lines indicate the least-square line fitting the data. Larger slopes indicate stronger center-surround interaction. Inset in the right shows an augmented version of the dotted box. (f) Tm9 responses to full field flashes to ON and OFF stimuli (2 s) interleaved by an intermediate stimulus background (4 s), in the presence (blue) and absence (orange) of CDM. Control $n=70, C D M ~ n=86$. Few cells show an increase in calcium signal in response to OFF. This inverted response type likely represents cells not directly looking at the screen (see also ${ }^{14}$ ). (g) Distribution of the mean response amplitude of Tm9 during the presentation of the OFF and ON components from data in (f). P-values from permutation tests are shown in the plot. (h-i) Physiological basis of $\mathrm{T}_{5}$ direction-selective receptive fields. (h) Voltage receptive fields of $\mathrm{T}_{5}$ from axon terminal recordings (from Fig. 1a, adapted from Wienecke et al. 2018). (i) Schematic showing receptive fields recorded using ON and OFF bars (Fig. 4, 5). The receptive field properties of Tm1, Tm2, Tm4 and $\mathrm{CT}_{1}$ can explain the receptive field structure of $\mathrm{T}_{5}$ from (Wienecke et al. 2018). (j) Schematic showing the sharpening of Tm9 ON and OFF receptive fields upon application of CDM, an octopamine agonist mimicking active behavioral states.

All OFF pathway Tm neurons were previously shown to respond positively to OFF stimuli. ${ }^{14,33,34,36}$ Here, we showed that Tm9, Tm2, and CT1 also respond negatively to ON stimuli. All Tm neurons are cholinergic and considered to provide excitatory input to $\mathrm{T}_{5}$, whereas $\mathrm{CT}_{1}$ neurons are GABAergic and likely inhibitory to $\mathrm{T}^{13,45}$ (Fig. $7 \mathbf{h}, \mathbf{i}$ ). The OFF excitation from Tm neurons and OFF inhibition from CT1 neurons could be enough to describe the nonlinear summation mechanism observed for dark bars. ${ }^{21}$ With the newly identified ON receptive field properties, all three dendritic input sites thus also receive $\mathrm{ON}$ information: from $\mathrm{Tm} 9$ on the distal part of the dendrite, from $\mathrm{Tm} 2$ centrally, and via $\mathrm{CT}_{1}$ at the basal site (Fig. 1 ). Because all subunits respond positively to OFF and negatively to $\mathrm{ON}, \mathrm{T}_{5}$ receives contrast-opponent inputs across its receptive field.

Thanks to the extensive computational, behavioral, anatomical, genetic, and physiological characterization of the fly visual system, the substrate of this important neural computation is now well understood. In particular, measuring physiological response properties of the involved neurons reveals how a linear mechanism of motion computation can be implemented. This exemplifies how a thorough understanding of the neuronal circuit elements is necessary to unravel the implementation of the diverse computations performed by the brain. 


\section{Negative ON responses in OFF pathway neurons}

Our work characterizes the response properties of the main input neurons to direction-selective $\mathrm{T}_{5}$ cells. The OFF-pathway neurons Tm9, Tm2, and CT1 display visual responses to ON stimuli and can thus implement a contrast-opponent mechanism for computing motion by providing the hitherto unknown $\mathrm{ON}$ input to direction-selective $\mathrm{T}_{5}$ neurons of the OFF pathway. Earlier difficulty in observing $\mathrm{ON}$ responses may be due to the relative nature of calcium imaging. While electrical recordings have a concrete reference voltage, calcium imaging is influenced by baseline fluorescence, which is affected by various factors, including sensor expression level and basal cellular calcium, and largely determines the dynamic range of detectable changes. Raising this baseline allowed the detection of response decrements using calcium imaging in previous studies looking at color and motion opponency. ${ }^{22,46}$ Here, our stimulus design favored the identification of calcium decrements in response to ON stimuli by raising the baseline calcium signal of OFF Tm neurons with a dark background. Interestingly, $\mathrm{T}_{5}$ did not show significant negative calcium responses to $\mathrm{ON}$ bars, even when using a dark background. This is consistent with the notion of a rectifying nonlinearity of voltage to calcium in $\mathrm{T}_{5} \cdot{ }^{7,17}$ While direction selectivity exists in $\mathrm{T}_{5}$ voltage responses, it is considerably higher in $\mathrm{T}_{5}$ calcium responses. This conundrum of distinguishing linear from nonlinear processing steps was also addressed in studies of cortical simple cells. Whereas direction selectivity of spiking responses is nonlinear, membrane voltage integration is a linear process that is then followed by a nonlinear spiking threshold that amplifies the initial voltage selectivity. ${ }^{47}$

\section{Harnessing variability through simultaneous measurements}

Our work shows that neuronal responses of an identified cell type can vary between cells within the visual system. To link $\mathrm{Tm}_{9}$ and $\mathrm{T}_{5}$ properties, simultaneous measurements of their responses are crucial because of the variable nature of the neural response properties. So far, response properties of one cell type have been considered to be stereotypic, and in general, responses of different cells of one cell type are averaged to describe a cell type's properties. ${ }^{10,14,32,34,38,48,49}$ In general, variability can be used to decorrelate information in the circuit and increase encoding efficiency by reducing the redundancy of information transmitted by a neuronal population. ${ }^{5}$ For example, visual signals from photoreceptors are decorrelated after passing through the retina, LGN, and V1, and this decorrelation is accompanied by increased encoding efficiency $\cdot{ }^{51-53}$

Variability within a single cell type can emerge from intrinsic differences in the transcriptional landscape. Current advances in single-cell sequencing technologies are opening up avenues to study variability within neurons that are strictly thought to belong to one cell type by anatomical and genetic means. Recent studies identified dorsal and ventral Tm9 subtypes, ${ }^{54,55}$ which could contribute to the large variability of Tm9 ON receptive field sizes reported here (Fig. 2). Furthermore, variations in synaptic connectivity within a cell type might also account for the physiological diversity of Tm9 responses. Detailed information about connectivity is often limited to information on single or only a few cells from individual flies. It is very intriguing that Tm9 has been shown to be the most variable when comparing connectivity between seven neighboring columns in the medulla. ${ }^{56}$ 


\section{ON and OFF inputs for direction selectivity persist across behavioral states}

Sensory systems have evolved to match the requirements of the surrounding environment. Thus, the mechanisms for motion detection must be able to accommodate the wide distribution of natural stimuli in the world, and the variation of the stimulus distribution caused by the behavioral state of the organism. An active behavioral state, such as walking or flying, comes with higher relative speeds of visual motion cues that the visual system needs to handle, and is known to modulate the temporal frequency tuning of visually guided behaviors. ${ }^{39,41,57}$ Octopamine signaling modulates the temporal response properties of neurons at each stage of visual processing from the lamina,$^{58}$ to $\mathrm{T}_{4} / \mathrm{T}_{5}$ neurons and their medulla input neurons, ${ }^{32,59}$ and then to wide field neurons downstream of $\mathrm{T}_{4} / \mathrm{T}_{5} \cdot{ }^{40,43}$ In vertebrates, locomotion modulates visual responses from the early visual system ${ }^{60}$ to V1 cortical neurons. ${ }^{61}$

In Drosophila, $\mathrm{T}_{5}$ neurons shift their temporal tuning to higher frequencies in the presence of $\mathrm{CDM}{ }^{32}$ which could now be explained by $\mathrm{T}_{5}$ receiving less OFF inputs from its slowest presynaptic partner Tm9, while maintaining similar suppression of Tm9 ON inputs. Furthermore, octopamine does not only act in the temporal domain, ${ }^{32,59}$ but our work shows that it also ensures spatially precise sampling (Fig. $7 \mathbf{j}$ ). Mimicking an active state in the fly led to a spatial sharpening of Tm9 ON and OFF receptive fields, and a reduction in amplitude for OFF receptive field centers, which enhanced center-surround interactions and consequently reduced responses to global stimuli. Together, these properties could ensure spatially and temporally precise sampling when the fly is flying or walking. Thus, the physiological basis for direction selectivity described here can perform across behavioral states by changing the neuronal properties to accommodate the varying stimulus statistics.

\section{Universality of contrast-opponent mechanisms for direction selectivity}

Our study in Drosophila reveals the neural substrate underlying a linear mechanism of direction selectivity, a solution found by neural circuits in a range of species. Linear mechanisms were first proposed to explain the motion responses of cortical simple cells, ${ }^{24}$ and primate motion perception. ${ }^{62}$ Simple cells in the primary visual cortex receive thousands of synaptic inputs coming from thalamic neurons in the LGN, as well as from other cortical neurons. Most LGN neurons exhibit receptive fields with a classical center-surround structure and a push-pull organization, ${ }^{63}$ i.e. stimuli of opposite contrast lead to synaptic inputs of opposite polarity. In the Hubel and Wiesel view, simple cells are direction selective because they receive inputs from several LGN neurons, leading to receptive fields with two elongated ON and OFF subunits. ${ }^{64}$ Recent evidence shows a V1 neuron receives spatially offset inputs from excitatory and inhibitory neuron populations in agreement with its direction selectivity. ${ }^{65}$ In carnivores and primates, the simple cell subunits also have a push-pull structure. ${ }^{27-29}$ Similarly, $T_{5}$ neurons show a contrast-opponent organization (Fig. 1 g), as do the Tm9, Tm2, and CT1 input neurons, although direct measurements of excitatory and inhibitory input currents would be needed to confirm a push-pull property. Our findings highlight the conservation of contrast opponency across the visual hierarchy in organisms as different as cat and Drosophila. Because $\mathrm{T}_{5}$ receives orders of magnitude fewer input synapses than cortical cells, and only a few cell types constitute the large majority of input synapses, ${ }^{13,25}$ we were able to map 
the detailed circuit structure, as well as the physiological contribution of identified cell types for a neural computation that is present in many organisms. Thus, the fly offers the opportunity to understand the minimal motifs behind linear mechanisms of direction selectivity.

\section{Acknowledgements}

We thank Carlotta Martelli, Julijana Gjorgjieva, and members of the Silies lab for discussions and critical comments on the manuscript. We are grateful to Aljoscha Nern and Michael Reiser for sharing fly stocks. This project has received funding from the European Research Council (ERC) under the European Union's Horizon 2020 research and innovation programme (grant agreement No 716512).

\section{Author contributions}

G.R.-T. and M.S. conceived the experiments. G.R.-T. conceived the model, performed the experiments and analyzed the data. G.R.-T. and M.S. wrote the paper.

\section{Methods}

\section{Fly husbandry and preparation.}

Drosophila melanogaster were raised on molasses-based food at $25^{\circ} \mathrm{C}$ and $55 \%$ humidity, on a $12: 12$ $\mathrm{h}$ light-dark cycle. Female flies, 1-7 days after eclosion, were used for all experiments.

To open an optical window to their brain, flies were first immobilized in an empty vial by cooling on ice. Flies were inserted in a sheet of stainless steel foil, such that the thorax protruded but the rest of the body, particularly the eyes, remained below the foil. The fly was fixed to the foil by UV-cured glue (Bondic) applied to the thorax and the left portion of the head. To expose the right optic lobe for imaging from above, the cuticle, fat bodies, and trachea were removed using razor blades and forceps under ice-cold, low-calcium saline. Following dissection, low-calcium saline was exchanged with calcium saline at room temperature. Saline for calcium imaging was composed of $103 \mathrm{mM} \mathrm{NaCl}, 3 \mathrm{mM} \mathrm{KCl}, 5 \mathrm{mM}$ TES, $1 \mathrm{mM} \mathrm{NaH} 2 \mathrm{PO}_{4}, 4 \mathrm{mM} \mathrm{MgCl}$, $1.5 \mathrm{mM} \mathrm{CaCl}$, $10 \mathrm{mM}$ trehalose, $10 \mathrm{mM}$ glucose, $7 \mathrm{mM}$ sucrose, and $26 \mathrm{mM} \mathrm{NaHCO}_{3}$ (no calcium, no sugars for low-calcium saline). The solution was bubbled with carbogen $\left(95 \% \mathrm{O}_{2}, 5 \% \mathrm{CO}_{2}\right)$ and continuously perfused the fly brain $(60-100 \mathrm{~mL} / \mathrm{h})$. 


\section{Genotypes}

- $w+/ w+; T m 9^{24 C 08}-L e x A$,lexAop-GCaMP $6 f /+;+/+$

- $w+/ w$-; Tm $9^{24 C 08}$-LexA,lexAop-GCaMP6f/Tm4-split-Gal4-AD;UAS-jRGECO1a/Tm4-split-Gal4$D B D$

- $w+/ w-;$ Tm1-split-Gal4-AD/+;Tm1-split-Gal4-DBD/UAS-GCaMP6f

- $w+/ w$-; Tm2-split-Gal4-AD/+;Tm2-split-Gal4-DBD/UAS-GCaMP6f

- w+/w-; Tm4-split-Gal4-AD/+;Tm4-split-Gal4-DBD/UAS-GCaMP6f

- $w+/ w-; C T 1^{R 65 E 11}-G a l_{4}-A D / U A S-G C a M P 6 f ; C T 1^{R 20 C 09}-G a l_{4}-D B D / U A S-G C a M P 6 f$

- $w+/ w+;$ T4/T5 $5^{R 59 E 08}$-LexA,lexAop-GCaMP6f;UAS-jRGECO1a/Tm $9^{24 C 08}-G_{4} l_{4}$

\section{Imaging}

In vivo calcium signals were recorded using a Bruker Investigator two-photon microscope (Bruker, Madison, WI, USA) coupled to a tunable laser (Spectraphysics Insight DS+) with an additional output fixed at $1040 \mathrm{~nm}$. The microscope was equipped with a $25 \mathrm{x} / 1.1$ water-immersion objective (Nikon, Minato, Japan). Laser excitation was tuned to $920 \mathrm{~nm}$ for GCaMP6f only measurements, and to $935 \mathrm{~nm}$ for dual-imaging experiments with both T5»GCaMP6f and Tm9»jRGECO1a. For dual imaging of Tm9»GCaMP6f and Tm4»jRGECO1a, the main laser output was tuned to $920 \mathrm{~nm}$, and $1040 \mathrm{~nm}$ excitation was additionally delivered. Typically less than $20 \mathrm{~mW}$ of excitation was delivered to the specimen, measured at the objective.

Emitted light was sent through a SP680 short-pass filter, a 560 lpxr dichroic filter (which separated green and red emission) and either a 525/70 or a 595/50 emission filter. PMT gain was set to $855 \mathrm{~V}$ for both channels. The microscope was controlled with the PrairieView software. Imaging rate was 8-12 Hz for most recordings, images of approx. 90x256 pixels were recorded, using an optical zoom of $8 \mathrm{x}$ to $10 \mathrm{x}$.

\section{Visual stimulation}

\section{Setup}

Stimuli were programmed in C++ using OpenGL, and projected using a LightCrafter 450o DLP (Texas Instruments, Dallas, TX, USA) with only blue LED illumination. Stimulus light was attenuated with a $482 / 18$ bandpass and ND1 filters, before reaching a rear projection screen. The screen measured 8 $\mathrm{cm} \times 8 \mathrm{~cm}$, subtending about $60 \mathrm{deg} \times 60 \mathrm{deg}$ (azimuth $\times$ elevation) of the right visual field of the fly. Stimuli were displayed at 6-bit pixel depth and at a frame rate of $300 \mathrm{~Hz}$, but the stimulus frame was updated at $100 \mathrm{~Hz}$. The stimulus frame parameters, including timestamps, were saved to disk together with the imaging data frame timestamps to allow for stimulus-imaging time alignment during data analysis. The stimulus and data acquisition computers were linked via a NI-DAQ USB-6211 device (National Instruments). 


\section{ON / OFF bars}

To measure spatial receptive fields, stimuli consisted of 5-degree bars. ON and OFF bars were $100 \%$ Michelson contrast: bright bars on a dark background and vice versa. Individual bar positions covered the screen in 2-degree shifts. For each trial, bar positions were shuffled to be presented in a pseudo-random order. A single bar was flashed at each position for $1 \mathrm{~s}$, with a $1 \mathrm{~s}$ interstimulus interval at which background was shown. In total, bars of a single polarity and orientation (horizontal or vertical) were presented for four to five trials each.

\section{Ternary noise}

To measure spatiotemporal receptive fields, stimuli consisted of ternary noise. Each stimulus frame consisted of 12 bars tiling the screen either horizontally or vertically. The bars were 5 degrees wide. For each frame, each of the 12 bars was assigned a black, white or gray contrast value with equal probability, and independent from the other bars. The contrast of the bars was updated every 100 ms. A $3 \mathrm{~s}$ gray background was shown before starting the ternary noise stimulus.

\section{Sine wave gratings}

To measure spatiotemporal frequency tuning, stimuli consisted of moving sine wave gratings. The gratings moved in the left-to-right direction, and had $100 \%$ Michelson contrast. Spatial and temporal frequency was drawn from a two-dimensional parameter grid. The grid was constructed using the spatial wavelengths $2,3.5,6,10,18$, and 32 degrees per cycle, and temporal frequencies $0.25,0.5,1.25,3,8$, and $16 \mathrm{~Hz}$. In total, 36 gratings were shown for a single stimulus trial in a pseudo-random order. Gratings were presented for $4 \mathrm{~s}$ with $4 \mathrm{~s}$ gray background between gratings. The stimulus was presented for five trials.

\section{Data analysis}

\section{Data processing}

Imaging time series were registered to compensate for within-plane motion of the specimen. Image registration used either cross-correlation alignment or RASL (robust alignment by sparse and low-rank decomposition). ${ }^{66}$ To align across the time series of the same recording session, each time series was first independently registered to obtain a within-time-series registration. Then, the mean of each independently registered time series was used to register all time series across the recording by applying the obtained global shift to all frames of the within-time-series registered frames.

Fluorescence time series $\mathrm{F}(\mathrm{t})$ were high-pass filtered with a cutoff period of $\approx 0.1 \mathrm{~Hz}$ (or 150 data frames). ROIs were selected manually following the stereotypical shapes of the recorded neuron types. Pixels were averaged within each ROI. The time series was normalized as $\frac{\Delta \mathrm{F}}{F_{0}}=\frac{\left(F-F_{0}\right)}{F_{0}}$, where the baseline fluorescene $F_{0}$ was chosen as the average fluorescence during all presentation of the 
background stimulus. To avoid strong signal fluctuations for recordings with baseline signal close to 0 , the mean fluorescence of the full time series $\bar{F}$ was added to the denominator $\frac{\Delta \mathrm{F}}{F_{0}}=\frac{\left(F-F_{0}\right)}{\left(F_{0}+\bar{F}\right)}$.

The time of the fluorescence signal was aligned to the time of stimulus presentation before trial averaging. To average signals acquired at slightly different frame rates, all time series were interpolated to a common frequency of $10 \mathrm{~Hz}$.

The response variability of the fluorescence time traces across stimulus repetitions was quantified using a response quality index. ${ }^{67}$ This index is the signal-to-noise ratio given by

$Q_{i}=\frac{\operatorname{var}\left[\langle F\rangle_{\text {trials }}\right]_{\text {time }}}{\left\langle\operatorname{var}[F]_{\text {time }}\right\rangle_{\text {trials }}}$,

which is the variance across time of the response trial average divided by the trial average of the variances across time. For identical responses across trials this index equals one, while for completely random responses with fixed variance the index is inversely proportional to the number of trials. A threshold value of 0.5 for the response quality index was used to select responding neurons independently of the stimulus structure (Fig. 2,4,5,7). For dual color imaging, we applied a lower response quality index of 0.3 due to a lower signal-to-noise ratio of the jRGECO1a calcium indicator (Fig. 6).

\section{Tuning curves}

Spatial receptive fields To quantify the receptive field position, width and amplitude, we obtained a tuning curve as follows. The $1 \mathrm{~s}$ response traces to (shuffled) bar positions were averaged across trials and sorted by spatial coordinates (Fig. S2, $_{2}$ ). Then, for each bar position the $1 \mathrm{~s}$ response was collapsed to a single response parameter, the response with the maximum absolute value. The resulting tuning curve (example Fig. 4 b-e) was then fitted to a single Gaussian curve of the form $f(x)=A \times \exp \left(\frac{-\left(x-x_{0}\right)^{2}}{w^{2}}\right)$. The amplitude is given by $A$, the position by $x_{0}$, and the width by $w$. The full width at half maximum (FWHM) is then calculated as FWHM $=2 w \sqrt{\log 2}$. To quantify center-surround receptive fields, the same procedure was followed but the function fitted to the tuning curve was a difference-of-Gaussians of the form $f(x)=A_{c} \exp \left(\frac{-\left(x-x_{0}\right)^{2}}{w_{c}{ }^{2}}\right)-A_{s} \exp \left(\frac{-\left(x-x_{0}\right)^{2}}{w_{s}{ }^{2}}\right)$. Both center and surround are constrained to the same spatial location $x_{0}$, but allowed to have different amplitudes $\left(A_{c, s}\right)$ and widths $\left(w_{c, s}\right)$. We constrained the difference-of-Gaussians fitting algorithm to solutions with $A_{c}>A_{s}$, and $A_{c, s}$ amplitudes below twice the amplitude range of the original data.

Gaussian fits of Tm9 neurons were considered for quantification if their goodness-of-fit $\left(r^{2}\right)$ was greater than 0.5 for OFF bars, and 0.2 for ON bars (Fig. 2). For data of different Tm neurons matched across stimuli neurons had to pass the same thresholds for both orientations (Fig. 4, 5). For the same dataset of Fig. 4, all tuning curves without any selection criteria are displayed in Fig. $\mathrm{S}_{4}$. For dual color $\mathrm{Tm}_{4}$ and $\mathrm{Tm} 9$ imaging, because $\mathrm{Tm}_{4}$ neurons did not respond to ON bars (Fig. S3), a threshold of 0.8 was set for OFF responses of both Tm9 and $\mathrm{Tm}_{4}$ (Fig. 3). For data in Fig. 6, fits to responses to OFF bars were considered for $r^{2}>0.25$ for both Tm9 and $\mathrm{T}_{5}$. For Tm9 recordings with and without CDM, where both center and surround of the response was analyzed, difference-of-Gaussian fits were applied only for neurons passing the response quality threshold 
for all conditions (Fig. 7, S6).

Spatiotemporal receptive fields were obtained from responses to ternary noise using the fluorescence-weighted average stimulus. The spatiotemporal receptive fields (linear filters) are given by $k(x, \tau)=\frac{1}{(T-\tau)} \cdot \sum_{t=\tau}^{T} r(t) s(x, t-\tau)$, where $r(t)$ is the $\frac{\Delta F}{F_{0}}$ signal, $\tau$ is the time window of the stimulus average, and $s(x, t)$ is the value of stimulus at position $x$ and time $t$. Since a linear filter can only be determined up to an arbitrary multiplicative constant, ${ }^{68}$ the spatiotemporal receptive field was normalized to have a unit Euclidean norm (arbitrary units). The spatiotemporal receptive field was further split into a spatial and temporal receptive field by taking one-dimensional slices along the respective dimensions from the point of maximum receptive field strength. The spatial receptive field was fitted to a Gaussian curve similarly to the bar receptive fields.

Spatiotemporal frequency tuning was calculated using the Fourier amplitude spectrum of the response for the frequency matching the temporal frequency of the grating. The tuning similarity across the simultaneously imaged neuron types was quantified as the Pearson correlation between the two-dimensional maps of each neuron pair.

\section{$\mathrm{T}_{5}$ dendrite model}

The model was coded in Python using the NEURON simulation software. The geometry of the fly eye was approximated by an hexagonal array consisting of a central point surrounded by six neighbors spaced by five degrees. The points in the array defined the position of the receptive fields from the medulla input neurons Tm1, Tm2, Tm4, Tm9, and CT1.

The spatial receptive field of the neurons consisted of a difference-of-Gaussians. The temporal component was a low-pass filter for $\mathrm{CT}_{1}$ and $\operatorname{Tm} 9\left(f_{L P}(t)=2 \tau^{-3 / 2} t e^{-t / \tau}\right)$, and a high-pass filter for $\operatorname{Tm} 1, \operatorname{Tm} 2$, and $\operatorname{Tm}_{4}\left(f_{H P}(t)=2 \tau^{-3 / 2}(t-\tau) e^{-t / \tau}\right)$. Parameters for the spatiotemporal receptive fields follow from 32 ; parameters for Tm9 were transferred to $\mathrm{CT}_{1}$, because $\mathrm{CT}_{1}$ receives the majority of inputs from Tm9.

A visual stimulus consisting of a sinewave grating was first transformed using the spatiotemporal receptive field into an input current to the medulla input neurons, The current was then converted into a conductance change using synaptic dynamics with two time constants (alpha synapse) taken from 20. This conductance was fed into a nonlinearity approximating the contrast selectivity of the neurons. The nonlinearity was either a linear function indicating ON and OFF responses (positive for OFF, negative for ON), or a rectified linear function indicating preference for only OFF responses (zero ON, positive for OFF). The conductance output after the nonlinearity was then offset by a fixed constant equal for all neurons, to prevent negative conductances in the range of contrast used in the simulations, and keep them in the unit range $[0,1]$. The resulting conductance is the input from each neuron to the $\mathrm{T}_{5}$ dendrite model, which is weighted proportionally to the synaptic connectivity given by 13 . Inputs from $\mathrm{Tm}_{1}, \mathrm{Tm}_{2}, \mathrm{Tm}_{4}$, and $\mathrm{Tm} 9$ were excitatory with reversal potential at o $\mathrm{mV}$, while inhibitory inputs from $\mathrm{CT}_{1}$ had a reversal potential of $-70 \mathrm{mV}$. The amplitude of the input conductances was $2.49 \cdot 10^{-5} \mu \mathrm{S}$ for excitation and $4 \cdot 98 \cdot 10^{-4} \mu \mathrm{S}$ for inhibition, reflecting the range of values in 20. Our only free parameter is the inhibition to excitation ratio (I/E), which controls the relative strength of excitatory and inhibitory synapses, set to 55 . The $\mathrm{T}_{5}$ dendrite was simulated as a passive cable with $13.3 \mu \mathrm{m}$ length and $0.2 \mu \mathrm{m}$ diameter. Using parameters from a $\mathrm{T}_{4}$ dendrite model, ${ }^{20}$ the passive cable had $100 \mathrm{Ohm} \cdot \mathrm{cm}$ axial resistance, 1 
$\mu F / \mathrm{cm}^{2}$ membrane capacitance, $1.03 \cdot 10^{-4} \mathrm{~S} / \mathrm{cm}^{2}$ membrane conductance, and $-65 \mathrm{mV}$ leak potential. The $\mathrm{T}_{5}$ dendrite received inputs as follows: $\mathrm{Tm} 9$ inputs from the trailing column were given close to the dendrite tip; $\mathrm{Tm}_{1}, \mathrm{Tm}_{2}$, and $\mathrm{Tm}_{4}$ inputs from central column were given close to the dendrite center, and $\mathrm{CT}_{1}$ inputs from the leading column were given near the dendrite base. Finally, voltage responses of $\mathrm{T}_{5}$ were measured from the dendrite base.

To calculate direction selectivity, we simulated responses to gratings moving in 16 different directions for $6 \mathrm{~s}$; the gratings had a temporal frequency of $1 \mathrm{~Hz}$ and a spatial frequency of $1 / 24$ cycles per degree. We quantified the dendrite response as the component of the Fourier amplitude spectrum at the frequency of the stimulus. The response amplitude and the direction of the stimulus can be represented as a vector in polar coordinates. This way we measured the directions selectivity index (DSI) as the amplitude of the sum of the response vector across motion directions, divided by the sum of the response amplitudes. If all vectors respond equally in all directions, the vector average will be zero, indicating no direction selectivity, and if only one direction elicits responses, then the DSI will be one.

This model draws parameters from 20 and 32 and simulates a simple $\mathrm{T}_{5}$ dendrite constrained by the properties of the input neurons. While the model in 20 fitted the parameters to match $\mathrm{T}_{4}$ data without any direct consideration of the properties of the input neurons, we expect the synaptic parameters to be a good approximation to the real biophysical properties of $\mathrm{T}_{5}$ dendrites yet to be characterized. To test the robustness of the model to the readout quantity, besides the first harmonic amplitude, we also quantified the maximum of the dendritic voltage response, as well as the mean of the dendritic calcium response (Fig. $\left.S_{1} \mathbf{c}-\mathbf{e}\right)$. The calcium response was obtained by squaring the positively-rectified voltage response $\left([\Delta \mathrm{v}]_{+}\right)^{2}{ }^{27,37}$ We tested how the direction selectivity is influenced by our free parameter, the I/E ratio (Fig. S $\mathbf{f}-\mathbf{g}$ ). Interestingly, the relative ordering of the models remains similar across the wide range of $\mathrm{I} / \mathrm{E}$ values tested. The only difference occurs when $\mathrm{I} / \mathrm{E}$ is so large that responses are mostly negative (I/E above 80). Thus, our conclusions are largely independent of the I/E paramater choice. Finally, to test how the models behave in different regions of the stimulus space we quantified direction selectivity across temporal frequencies for the models in Fig. 1, Sic-e. The all linear model has consistently higher direction selectivity across the temporal frequency range about $1 \mathrm{~Hz}$, and across response readouts (Fig. $\left.S_{1} \mathbf{i}-\mathbf{k}\right)$. The all rectified model has comparable performance only for the voltage Fourier response component (Fig. $\left.S_{1} \mathbf{i}\right)$. Interestingly, the Tm9 and T2 linear model achieve similar performance to the all linear model in the mean calcium readout (Fig. $S_{1} \mathbf{k}$ ). Therefore, linear inputs benefit models of direction selectivity over a region of the parameter and stimulus space.

\section{Statistical analysis}

Nested permutation tests were used for statistical comparisons in Figs. 2, 7 to account for the potential correlations of neurons within the same fly. This was done by permuting all neurons from the same fly together rather than permuting neurons independently from each other. In Fig. 2f, non-nested permutations were used, since the number of neurons per fly was low to contribute to within fly variability. In Fig. $6 \mathbf{f}$, the Pearson's correlation and corresponding p-value were given by the corrcoef MATLAB command. And in Fig. $6 \mathbf{h}$, the significance of the correlation between the spatio-temporal frequency tuning maps of pairs of overlapping $\mathrm{Tm}_{9}$ and $\mathrm{T}_{5}$ neurons was calculated as follows. The Tm9 and $\mathrm{T}_{5}$ pairs were randomly shuffled, the pairwise correlations 
were calculated, and the mean of this shuffled distribution was computed. The process was repeated 1e6 times resulting in a shuffled control distribution. A p-value was calculated as the ratio of mean shuffled correlations larger than the original mean correlation to the total number of shuffles (1e6). A p-value of o was obtained since no mean shuffle was ever higher than the original mean. In Fig. $7 \mathbf{e}$, the Pearson's correlations and their 95\%-confidence intervals were computed by 1000 bootstrap runs.

\section{Data availability}

The data presented here is available upon reasonable request to the corresponding author.

\section{Software availability}

Software for the model is deposited at https://github.com/silieslab/RamosTraslosheros_Silies_2020

\section{References}

1. Clark, D. A. \& Demb, J. B. Parallel Computations in Insect and Mammalian Visual Motion Processing. Current Biology 26, R1062-R1072 (2016).

2. Mauss, A. S., Vlasits, A., Borst, A. \& Feller, M. Visual Circuits for Direction Selectivity. Annual Review of Neuroscience 40, 211-230 (2017).

3. Ramos-Traslosheros, G., Henning, M. \& Silies, M. Motion detection: Cells, circuits and algorithms. Neuroforum 24, A61-A72 (2018).

4. Silies, M., Gohl, D. M. \& Clandinin, T. R. Motion-Detecting Circuits in Flies: Coming into View. Annual Review of Neuroscience 37, 307-327 (2014).

5. Takemura, S.-y. Connectome of the fly visual circuitry. Microscopy 64, 37-44 (2015).

6. Yang, H. H. \& Clandinin, T. R. Elementary Motion Detection in Drosophila: Algorithms and Mechanisms. Annual Review of Vision Science 4, 143-163 (2018).

7. Wienecke, C. F. R., Leong, J. C. S. \& Clandinin, T. R. Linear Summation Underlies Direction Selectivity in Drosophila. Neuron 99, 680-688.e4 (2018).

8. Fisher, Y. E., Silies, M. \& Clandinin, T. R. Orientation Selectivity Sharpens Motion Detection in Drosophila. Neuron 88, 390-402 (2015).

9. Maisak, M. S. et al. A directional tuning map of Drosophila elementary motion detectors. Nature 500, 212-216 (2013).

10. Strother, J. A. et al. The Emergence of Directional Selectivity in the Visual Motion Pathway of Drosophila. Neuron 94, 168-182.e10 (2017). 
11. Takemura, S.-y. et al. The comprehensive connectome of a neural substrate for 'ON' motion detection in Drosophila. eLife 6, e24394 (2017).

12. Shinomiya, K. et al. Candidate Neural Substrates for Off-Edge Motion Detection in Drosophila. Current Biology 24, 1062-1070 (2014).

13. Shinomiya, K. et al. Comparisons between the ON- and OFF-edge motion pathways in the Drosophila brain. eLife 8, e40025 (2019).

14. Fisher, Y. E. et al. A Class of Visual Neurons with Wide-Field Properties Is Required for Local Motion Detection. Current Biology (2015).

15. Haag, J., Mishra, A. \& Borst, A. A common directional tuning mechanism of Drosophila motion-sensing neurons in the $\mathrm{ON}$ and in the OFF pathway. elife 6, e29044 (2017).

16. Haag, J., Arenz, A., Serbe, E., Gabbiani, F. \& Borst, A. Complementary mechanisms create direction selectivity in the fly. eLife 5, e17421 (2016).

17. Leong, J. C. S., Esch, J. J., Poole, B., Ganguli, S. \& Clandinin, T. R. Direction Selectivity in Drosophila Emerges from Preferred-Direction Enhancement and Null-Direction Suppression. fournal of Neuroscience 36, 8078-8092 (2016).

18. Hassenstein, B. \& Reichardt, W. Systemtheoretische Analyse der Zeit-, Reihenfolgen- und Vorzeichenauswertung bei der Bewegungsperzeption des Rüsselkäfers Chlorophanus. Zeitschrift für Naturforschung B 11, 513-524 (1956).

19. Barlow, H. B. \& Levick, W. R. The mechanism of directionally selective units in rabbit's retina. The fournal of Physiology 178, 477-504 (1965).

20. Gruntman, E., Romani, S. \& Reiser, M. B. Simple integration of fast excitation and offset, delayed inhibition computes directional selectivity in Drosophila. Nat. Neurosci. 21, 250-257 (2018).

21. Gruntman, E., Romani, S. \& Reiser, M. B. The computation of directional selectivity in the Drosophila OFF motion pathway. eLife 8, e50706 (2019).

22. Badwan, B. A., Creamer, M. S., Zavatone-Veth, J. A. \& Clark, D. A. Dynamic nonlinearities enable direction opponency in Drosophila elementary motion detectors. Nature Neuroscience 22, 1318-1326 (2019).

23. Jagadeesh, B., Wheat, H. \& Ferster, D. Jagadeesh, B., Wheat, H. S. \& Ferster, D. Linearity of summation of synaptic potentials underlying direction sleectivity in simple cells of the cat visual cortex. Science 262, 1901-1904. Science (New York, N.Y.) 262, 1901-4 (1994).

24. Jagadeesh, B., Wheat, H. S., Kontsevich, L. L., Tyler, C. W. \& Ferster, D. Direction selectivity of synaptic potentials in simple cells of the cat visual cortex. F Neurophysiol 78, 2772-2789 (1997).

25. Lien, A. D. \& Scanziani, M. Cortical direction selectivity emerges at convergence of thalamic synapses. Nature 558, 80-86 (2018).

26. Livingstone, M. S. Mechanisms of Direction Selectivity in Macaque V1. Neuron 20, 509-526 (1998). 
27. Jones, J. P. \& Palmer, L. A. The two-dimensional spatial structure of simple receptive fields in cat striate cortex. Journal of Neurophysiology 58, 1187-1211 (1987).

28. Ferster, D. Spatially opponent excitation and inhibition in simple cells of the cat visual cortex. $\mathcal{F}$. Neurosci. 8, 1172-1180 (1988).

29. Hirsch, J. A., Alonso, J.-M., Reid, R. C. \& Martinez, L. M. Synaptic Integration in Striate Cortical Simple Cells. f. Neurosci. 18, 9517-9528 (1998).

30. Salazar-Gatzimas, E. et al. Direct Measurement of Correlation Responses in Drosophila Elementary Motion Detectors Reveals Fast Timescale Tuning. Neuron 92, 227-239 (2016).

31. Salazar-Gatzimas, E., Agrochao, M., Fitzgerald, J. E. \& Clark, D. A. The Neuronal Basis of an Illusory Motion Percept Is Explained by Decorrelation of Parallel Motion Pathways. Current Biology 28, 3748-3762.e8 (2018).

32. Arenz, A., Drews, M. S., Richter, F. G., Ammer, G. \& Borst, A. The Temporal Tuning of the Drosophila Motion Detectors Is Determined by the Dynamics of Their Input Elements. Current Biology 27, 929-944 (2017).

33. Behnia, R., Clark, D. A., Carter, A. G., Clandinin, T. R. \& Desplan, C. Processing properties of ON and OFF pathways for Drosophila motion detection. Nature 512, 427-430 (2014).

34. Serbe, E., Meier, M., Leonhardt, A. \& Borst, A. Comprehensive Characterization of the Major Presynaptic Elements to the Drosophila OFF Motion Detector. Neuron (2016).

35. Strother, J. A., Nern, A. \& Reiser, M. B. Direct Observation of ON and OFF Pathways in the Drosophila Visual System. Current Biology 24, 976-983 (2014).

36. Meier, M. \& Borst, A. Extreme Compartmentalization in a Drosophila Amacrine Cell. Current Biology 29, 1545-1550.e2 (2019).

37. Zavatone-Veth, J. A., Badwan, B. A. \& Clark, D. A. A minimal synaptic model for direction selective neurons in Drosophila. Journal of Vision 20, 2 (2020).

38. Ketkar, M. D. et al. Luminance Information Is Required for the Accurate Estimation of Contrast in Rapidly Changing Visual Contexts. Current Biology o, (2020).

39. Chiappe, M. E., Seelig, J. D., Reiser, M. B. \& Jayaraman, V. Walking Modulates Speed Sensitivity in Drosophila Motion Vision. Current Biology 2o, 1470-1475 (2010).

40. Suver, M. P., Mamiya, A. \& Dickinson, M. H. Octopamine Neurons Mediate Flight-Induced Modulation of Visual Processing in Drosophila. Current Biology 22, 2294-2302 (2012).

41. Maimon, G., Straw, A. D. \& Dickinson, M. H. Active flight increases the gain of visual motion processing in Drosophila. Nature Neuroscience 13, 393-399 (2010).

42. Jung, S. N., Borst, A. \& Haag, J. Flight Activity Alters Velocity Tuning of Fly Motion-Sensitive Neurons. Journal of Neuroscience 31, 9231-9237 (2011).

43. Longden, K. D. \& Krapp, H. G. Octopaminergic Modulation of Temporal Frequency Coding in an Identified Optic Flow-Processing Interneuron. Frontiers in Systems Neuroscience 4, (2010). 
44. Agrochao, M., Tanaka, R., Salazar-Gatzimas, E. \& Clark, D. A. Mechanism for analogous illusory motion perception in flies and humans. Proceedings of the National Academy of Sciences 117, 23044-23053 (2020).

45. Davis, F. P. et al. A genetic, genomic, and computational resource for exploring neural circuit function. eLife 9, e50901 (2020).

46. Schnaitmann, C. et al. Color Processing in the Early Visual System of Drosophila. Cell 172, 318-330.e18 (2018).

47. Priebe, N. J. \& Ferster, D. Direction selectivity of excitation and inhibition in simple cells of the cat primary visual cortex. Neuron 45, 133-145 (2005).

48. Molina-Obando, S. et al. ON selectivity in the Drosophila visual system is a multisynaptic process involving both glutamatergic and GABAergic inhibition. eLife 8, e49373 (2019).

49. Silies, M. et al. Modular Use of Peripheral Input Channels Tunes Motion-Detecting Circuitry. Neuron 79, 111-127 (2013).

50. Barlow, H. Possible principles underlying the transformations of sensory messages (pp. 217). (1961).

51. Dan, Y., Atick, J. J. \& Reid, R. C. Efficient Coding of Natural Scenes in the Lateral Geniculate Nucleus: Experimental Test of a Computational Theory. Fournal of Neuroscience 16, 3351-3362 (1996).

52. Franke, K. et al. Inhibition decorrelates visual feature representations in the inner retina. Nature 542, 439-444 (2017).

53. Vinje, W. E. Sparse Coding and Decorrelation in Primary Visual Cortex During Natural Vision. Science 287, 1273-1276 (2000).

54. Özel, M. N. et al. Neuronal diversity and convergence in a visual system developmental atlas. Nature 589, 88-95 (2021).

55. Kurmangaliyev, Y. Z., Yoo, J., Valdes-Aleman, J., Sanfilippo, P. \& Zipursky, S. L. Transcriptional Programs of Circuit Assembly in the Drosophila Visual System. Neuron 108, 1045-1057.e6 (2020).

56. Takemura, S.-y. et al. Synaptic circuits and their variations within different columns in the visual system of Drosophila. Proceedings of the National Academy of Sciences 112, 13711-13716 (2015).

57. Maimon, G. Modulation of visual physiology by behavioral state in monkeys, mice, and flies. Current Opinion in Neurobiology 21, 559-564 (2011).

58. Tuthill, J. C., Nern, A., Rubin, G. M. \& Reiser, M. B. Wide-Field Feedback Neurons Dynamically Tune Early Visual Processing. Neuron 82, 887-895 (2014).

59. Strother, J. A. et al. Behavioral state modulates the ON visual motion pathway of Drosophila. Proceedings of the National Academy of Sciences 115, E102-E111 (2018). 
6o. Erisken, S. et al. Effects of Locomotion Extend throughout the Mouse Early Visual System. Current Biology 24, 2899-2907 (2014).

61. Niell, C. M. \& Stryker, M. P. Modulation of Visual Responses by Behavioral State in Mouse Visual Cortex. Neuron 65, 472-479 (2010).

62. Adelson, E. H. \& Bergen, J. R. Spatiotemporal energy models for the perception of motion. fournal of the Optical Society of America A 2, 284 (1985).

63. Suresh, V. et al. Synaptic Contributions to Receptive Field Structure and Response Properties in the Rodent Lateral Geniculate Nucleus of the Thalamus. Fournal of Neuroscience 36, 10949-10963 (2016).

64. Hubel, D. H. \& Wiesel, T. N. Receptive fields, binocular interaction and functional architecture in the cat's visual cortex. The fournal of Physiology 160, 106-154 (1962).

65. Rossi, L. F., Harris, K. D. \& Carandini, M. Spatial connectivity matches direction selectivity in visual cortex. Nature (2020).

66. Peng, Y., Ganesh, A., Wright, J., Xu, W. \& Ma, Y. RASL: Robust alignment by sparse and low-rank decomposition for linearly correlated images. IEEE transactions on pattern analysis and machine intelligence 34, 2233-2246 (2012).

67. Baden, T. et al. The functional diversity of retinal ganglion cells in the mouse. Nature 529, 345-350 (2016).

68. Chichilnisky, E. J. A simple white noise analysis of neuronal light responses. Network: Computation in Neural Systems 12, 199-213 (2001). 


\section{Supplements}

\section{Supplementary figures}

a

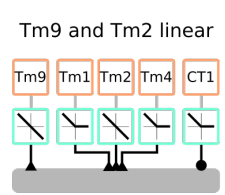

b

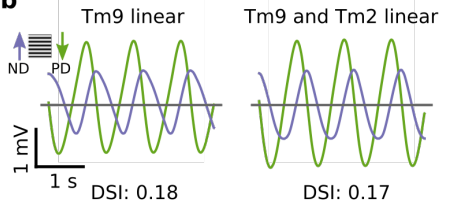

C

d $\quad$ DS-tuning $(\max (\Delta \mathrm{v}))$

e

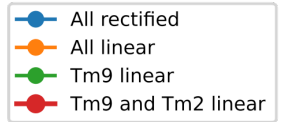

DS-tuning $(\mathrm{a} 1(\Delta \mathrm{v}))$

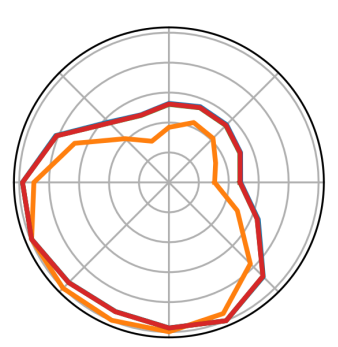

DS-tuning (mean $\left.\left([\Delta v]_{+}\right)^{2}\right)$
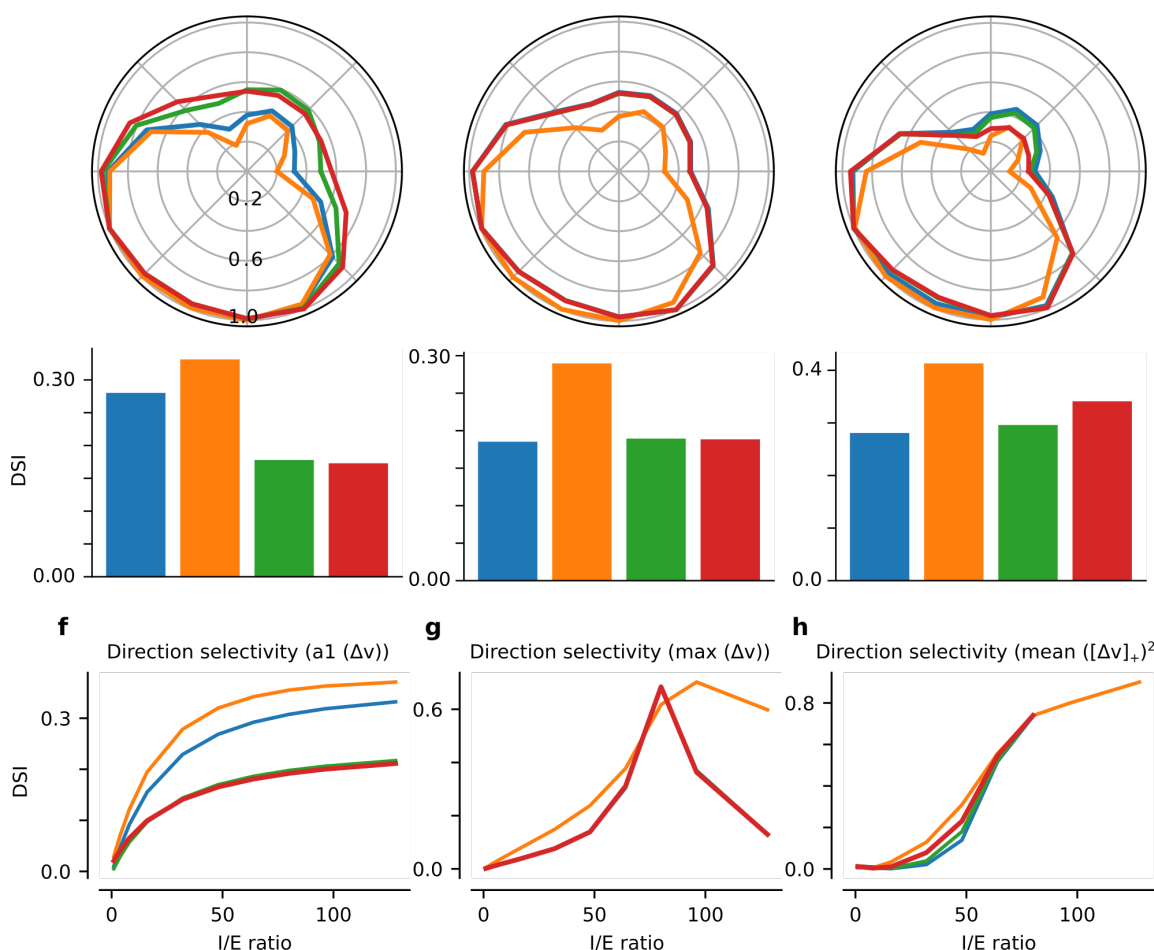

g

h

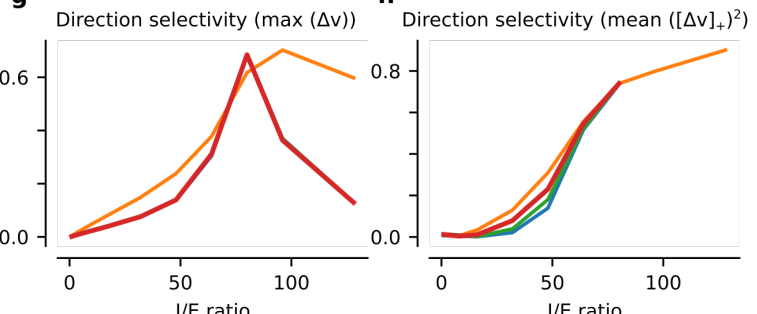

i
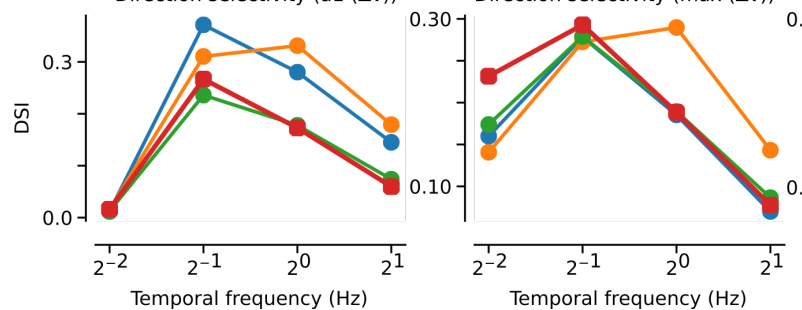

Direction selectivity (mean $\left.\left([\Delta v]_{+}\right)^{2}\right)$

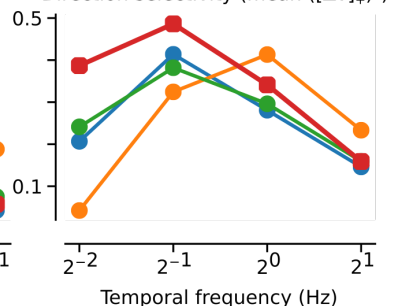

Figure S1: Comparison of models of local motion detection in Drosophila across readouts, parameters, and temporal frequencies. Continued on next page. 
Figure S1: Comparison of models of local motion detection in Drosophila across readouts, parameters, and temporal frequencies. (a) Schematic showing a $\mathrm{T}_{5}$ model with a linear OFF input on the Tm9, and the Tm2 sites. (b) Voltage responses in the preferred and null directions (ND) to a sinewave grating from the Tm9 linear model (Fig. 1 g), and the Tm9 and Tm2 linear model in (a). The direction-selectivity index (DSI) is shown below the traces, calculated from the amplitude of the Fourier component at $1 \mathrm{~Hz}$ (a1 amplitude) of the voltage responses for each of the 16 directions of motion simulated. Model responses are to a grating with a spatial frequency of $24 \mathrm{deg} /$ cycle and temporal frequency of $1 \mathrm{~Hz}(\mathbf{b}-\mathbf{h})$. (c-e) Tuning curves across motion directions for models in (Fig. 1 f) and (a). Top: Polar plots of response vs. direction of motion for the a1 amplitude of the voltage response (c), the maximum voltage response (d), and the mean calcium response (e). The calcium response was obtained by squaring the positively-rectified voltage response $\left([\Delta \mathrm{v}]_{+}\right)^{2}$. Bottom: Direction-selectivity indices obtained from the tuning curves on the corresponding panels on top. (f-h) Direction-selectivity index vs. inhibition to excitation ratio (I/E) quantified using the a 1 amplitude of the voltage response (f), the maximum voltage response (g), and the mean calcium response (h). For large I/E (above 80) responses are mostly negative, resulting in no calcium response. The all linear model consistently achieves higher DSI than the rest. (i-k) Direction-selectivity index vs. temporal frequency quantified using the a1 amplitude of the voltage response (i), the maximum voltage response $(\mathbf{j})$, and the mean calcium response (k). Model responses are to a grating with a spatial frequency of $24 \mathrm{deg} / \mathrm{cycle}$, moving downwards with temporal frequencies of $0.25 \mathrm{~Hz}, 0.5 \mathrm{~Hz}, 1 \mathrm{~Hz}$, and $2 \mathrm{~Hz}$. The all linear or the Tm9 and Tm2 linear models consistently achieves higher DSI than the rest. Linear inputs benefit models of direction selectivity over a region of the parameter and stimulus space.
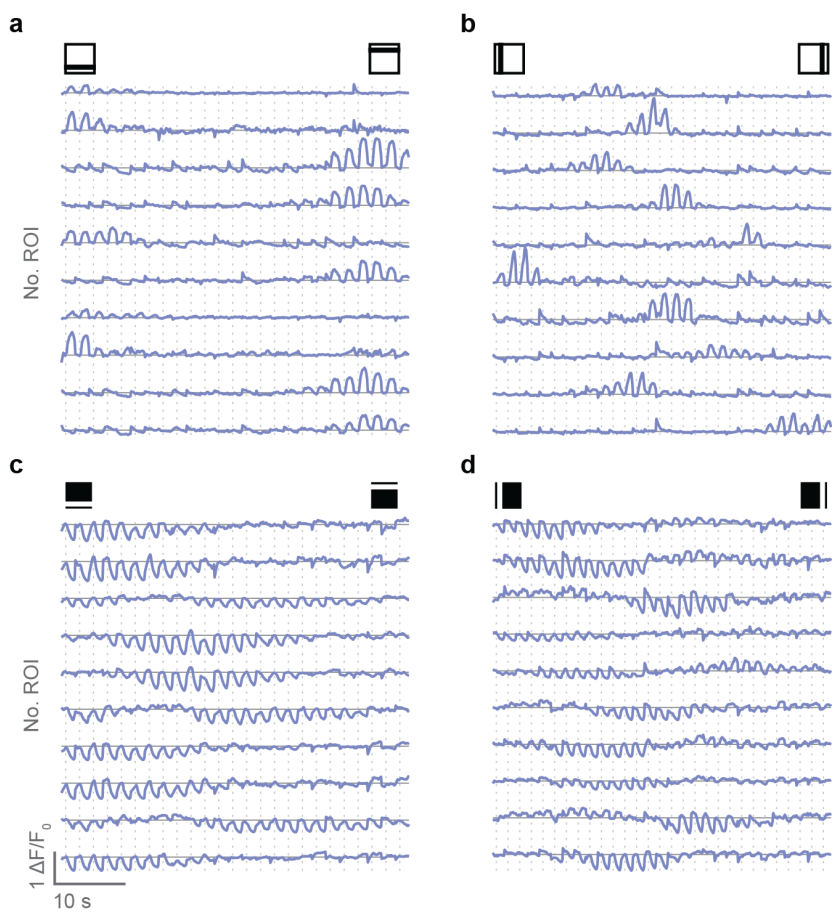

Figure S2: Tm9 calcium traces. Calcium traces of ten Tm9 example neurons to horizontal and vertical OFF $(\mathbf{a}, \mathbf{b})$ or ON (c,d) bars. Neurons from the dataset shown in (Fig. 2) were randomly selected for each stimulus. For each bar position, the calcium response ranging from $0.5 \mathrm{~s}$ before the appearance of the bar $(1 \mathrm{~s})$ to $0.5 \mathrm{~s}$ after the disappearance of the bar was trial averaged. Calcium traces are concatenated matching the spatial order of the bar. In the stimulus presentation, the order of the bar presentations was pseudo-randomized. 
$\mathbf{a}$

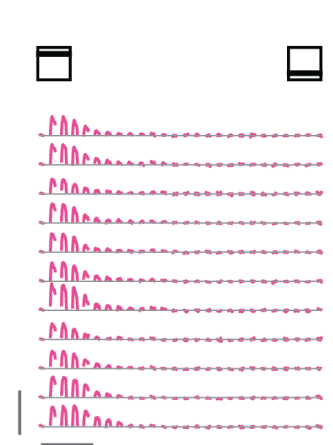

c

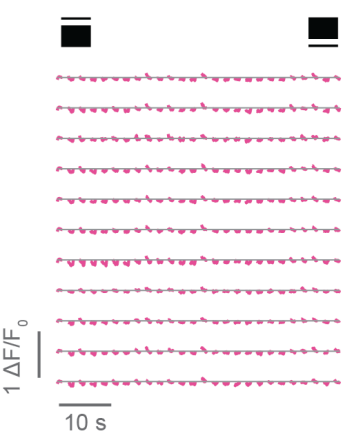

$\operatorname{Tm} 4$

\section{$\square$}
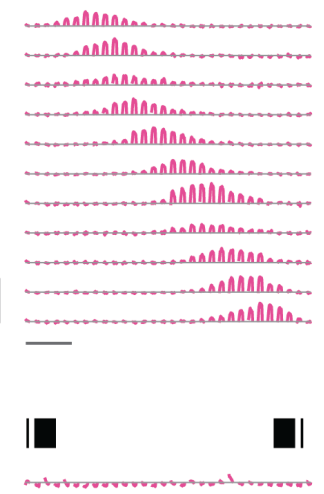

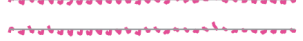

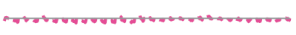

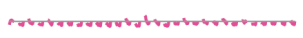

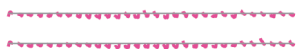

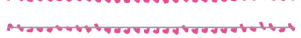

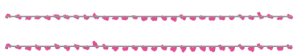

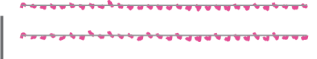

b

$\operatorname{Tm} 9$

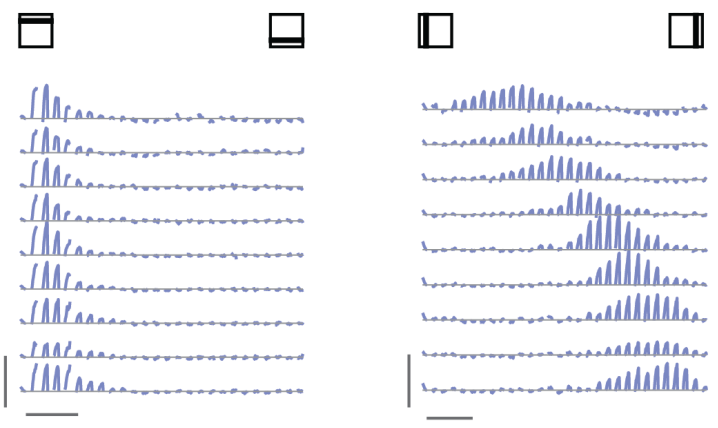

d

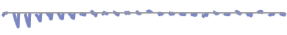

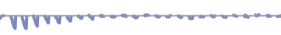

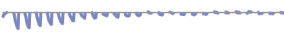

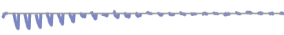

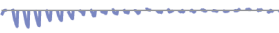

There

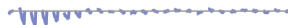

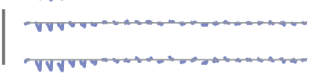

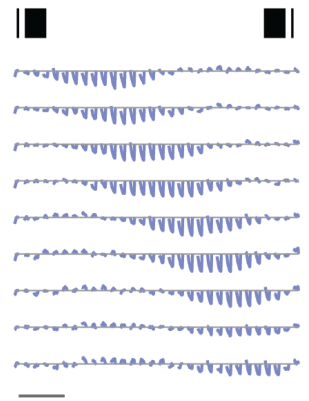

Figure S3: Tm9 and $\operatorname{Tm}_{4}$ calcium traces from the same fly. Calcium traces of $\operatorname{Tm}_{4}(\mathbf{a}, \mathbf{c})$ and $\operatorname{Tm} 9(\mathbf{b}, \mathbf{d})$ example neurons imaged in one fly, responding to horizontal and vertical OFF (a,b) or ON (c,d) bars. For each bar position, the calcium response during the $1 \mathrm{~s}$ presentation of the bar was trial averaged. Calcium traces are concatenated matching the spatial order of the bar. In the stimulus presentation, the order of the bar presentations was pseudo-randomized. 

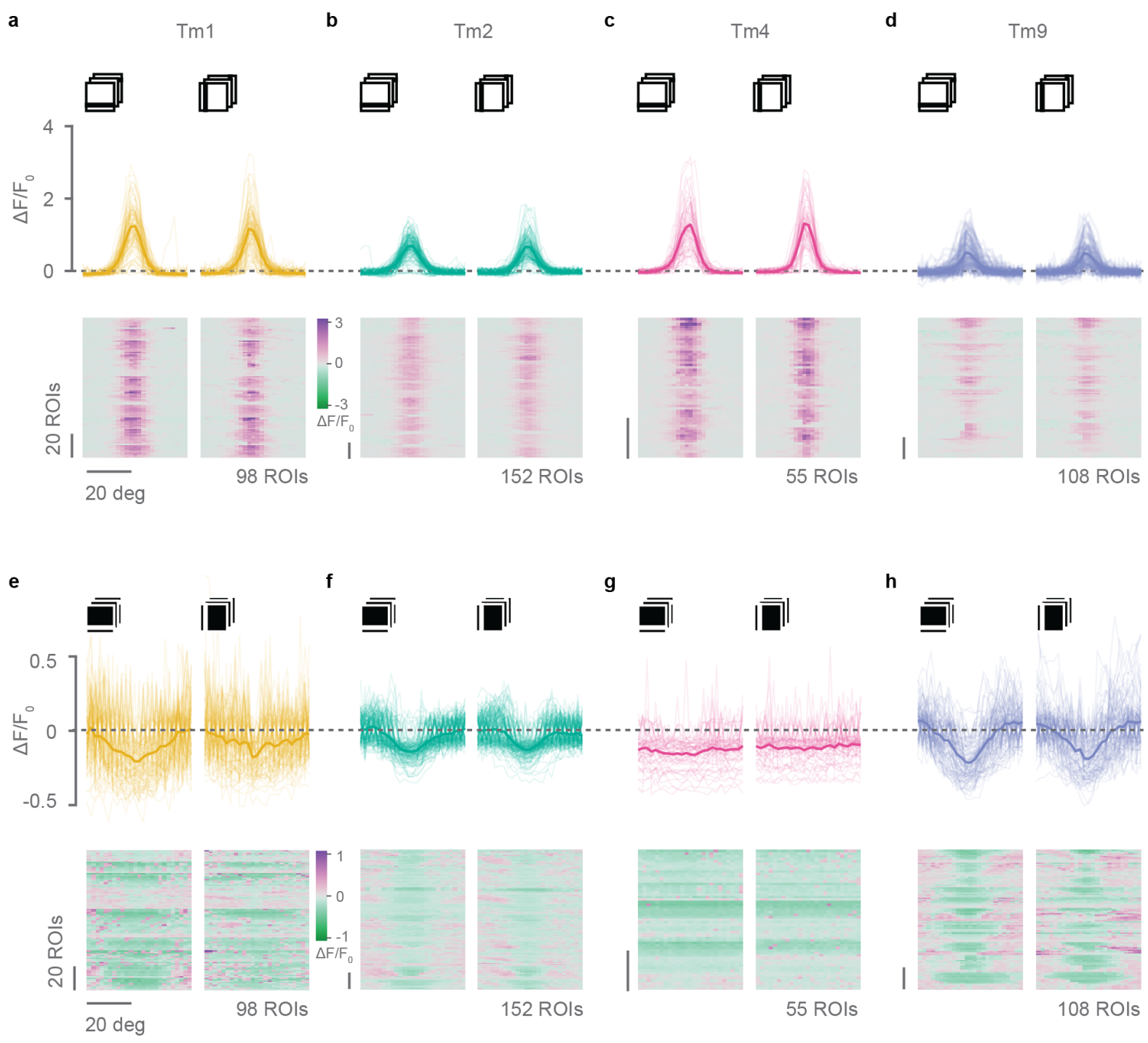

Figure S4: Receptive fields of Tm1, Tm2, Tm4 and Tm9 neurons. (a-d) OFF receptive fields obtained from in vivo two-photon calcium imaging of responses to vertical and horizontal dark bars from Tm1 (a), $\mathrm{Tm}_{2}(\mathbf{b}), \mathrm{Tm}_{4}$ (c), and Tm9 (d) neurons expressing GCaMP6f. This shows all recorded neurons, from which a subset with responses in both orientations fitted by a single Gaussian with $r^{2}>0.2$ were included in (Fig. 4). Number of ROIs (neurons) is shown below the plots on the right. (e-h) ON receptive fields obtained from responses to vertical and horizontal bright bars from $\operatorname{Tm} 1(\mathbf{e}), \operatorname{Tm} 2(\mathbf{f}), \operatorname{Tm} 4(\mathbf{g})$, or $\operatorname{Tm} 9$ (h) neurons in (a-d). Only $\mathrm{Tm} 2$ and Tm9 neurons had ON receptive fields visible averaging all recorded neurons without any selection criteria. This shows all recorded neurons, from which a subset with responses in both orientations fitted by a single Gaussian with $r^{2}>0.2$ were included in (Fig. 4 ). 
$\mathbf{a}$
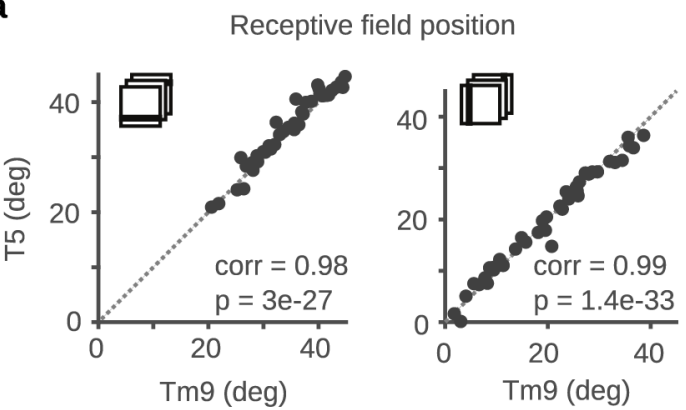

b

RF Pos. Difference (Tm9 - T5)
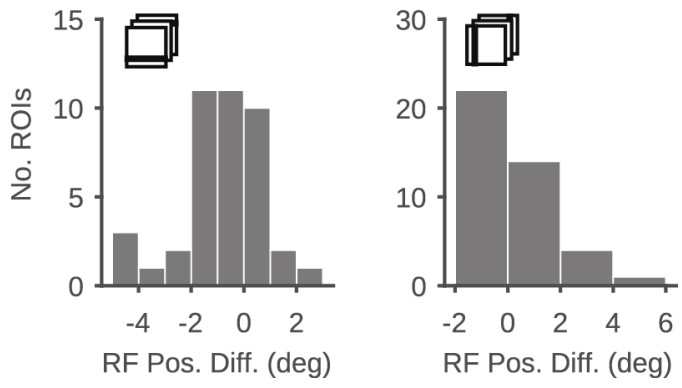

Figure $S_{5}$ : Receptive field positions are closely matched for overlapping $\mathbf{T}_{5}$ and Tm9 ROIs. (a) Scatter plot of receptive field positions obtained from Gaussian fits to $\mathrm{Tm} 9$ and $\mathrm{T}_{5}$ tuning curves in response to OFF bars shown in (Fig. 6b,d). Pearson's correlation are significant. (b) Distribution of receptive field position differences between overlapping $\mathrm{Tm}_{9}$ and $\mathrm{T}_{5}$ ROIs in (a), showing receptive fields overlap in visual space. 
Non-parametric amplitude quantification

a

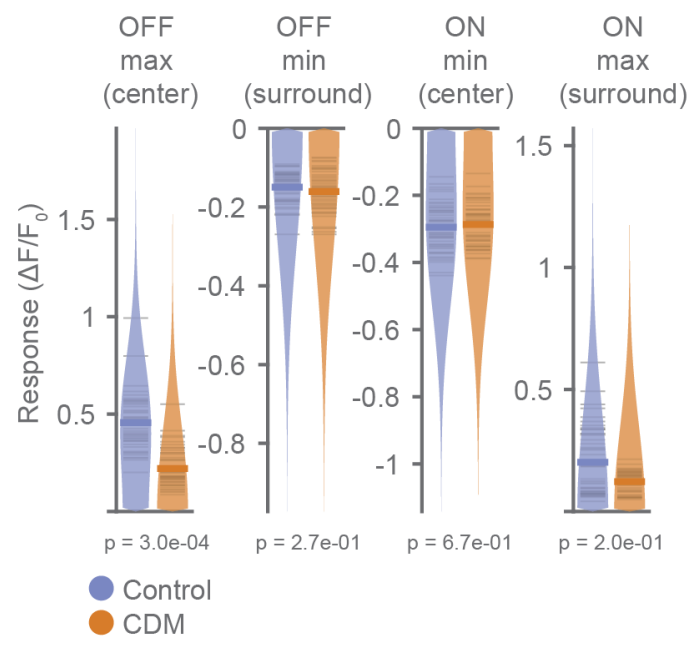

RF size from single Gaussian fit

b

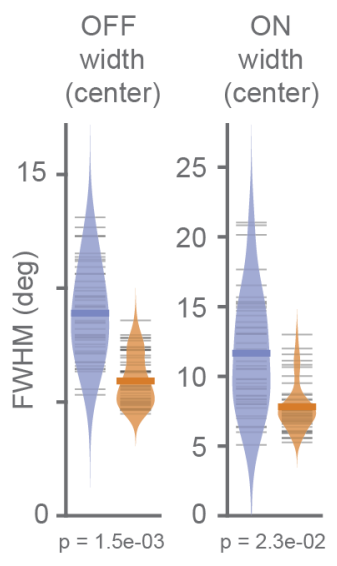

Figure S6: CDM effects on Tm9 receptive fields. (a) Non-parametric quantification of the approximate receptive field center and surround amplitudes, respectively defined by the maximum and minimum values for positive OFF receptive fields, and as the minimum and maximum responses for the negative ON receptive fields. Data points correspond to the ones used in (Fig. $7 \mathbf{a}$-e). (b) Receptive field full width at half maximum (FWHM) obtained from a single Gaussians fit (to data in (Fig. $7 \mathbf{a}, \mathbf{b}$ ). To quantify size changes of the net receptive fields. Each point is the average of the minimum/maximum amplitude (a) or fit FWHM (b) of a neuron across orientations. Two-tailed permutation tests comparing controls and CDM, p-values are indicated. 